\title{
GUIDANCE DOCUMENT ON THE VARIANCE PROPAGATION REQUIREMENTS IN DOE ORDER 5633.3
}

Jonathan Sanborn

\author{
October 1989 \\ (Revised October 1991)
}

TECHNICAL SUPPORT ORGANIZATION

DEPARTMENT OF NUCLEAR ENERGY

BROOKHAVEN NATIONAL LABORATORY, ASSOCIATED UNIVERSITIES. INC.

UPTON, NEW YORK 11973

Prepared for the

OFFICE OF SAFEGUARDS AND SECURITY

UNITED STATES DEPARTMENT OF ENERGY

WASHINGTON, D.C. 20545 


\section{DISCLAIMER}

This report was prepared as an account of work sponsored by an agency of the United States Government. Neither the United States Government nor any agency thereof. nor any of their employecs, nor any of their contractors. subcontractors, or their employees, makes any warranty, express or implied, or assumes any legal liability or responsibility for the accuracy, complateness, or usefulness of any information. apparatus, product, or process disclosed, or represents that its use would not infringe privately owned rights. Reference herein to any specific commercial product. process, or service by trade name, trademark, manufacturer, or otherwise, does not necesaarily conatitute or imply its endorsement. recommendation, or favoring by the United States

Government or any agency, contractor or subcontractor thereof. The views and opinions of authors expressed herein do not necessarily state or reflect those of the United States Government or any agency, contractor or subcontractor thereof.

Printed in the United States of America

Available from

National Technical Information Service

U.S. Department of Commerce

5285 Port Royal Road

Springfield, VA 22161

NTIS price codes:

Printed Copy: A05; Microfiche Copy: A01 
DOE facilities handling special nuclear material are required to comply with DOE Order 5633.3, which addresses various aspects of nuclear material accounting. In particular, it requires that control limits be calcula ed for nuclear material inventory differences. These requirements and methods of meeting them are described. The four steps in this process are the creation of a variance propagation model of the nuclear material accounting system, the validation of the model, the application of the model, and the documentation of the model; the first two of these steps are discussed at length. Topics addressed include: the mathematics of variance propagation, the structure of nuclear material accounting systems, sources of uncertainty in real accounting systems, statistical models of these sources of uncertainty, methods of estimating error standard deviations, calculation of control limits from inventory difference standard deviations, and comparison of models with historical inventory difference data. A chapter is also devoted to calculational methods. The approach emphasizes constructing models that can be implemented relatively easily and improved as data is accumulated and understanding of the system is gained, rather than attempting all-inclusive, highly detailed models at the outset. 


\section{ACKNOWLEDGMENTS}

The author is indebted to Mr. Joseph Rivers, formerly of DOE/OSS for many clarifying suggestions, and to Messrs. James Lemley and Theodor Teichmann, of BNL/TSO for help in preparing the manuscript.

$$
-i v \text { - }
$$




\section{CONTENTS}

I. Introduction 1

II. The Variance Propagation Requirements of DOE Order 5633.3 3

A. The Scope of the Requirement 3

B. An Overview 7

C. Modelling Real Accounting Systems $\quad 10$

D. Computing Control Limits 15

1. Long Term Trends 15

2. Correlation Between Successive IDs 15

III. Basic Variance Propagation Concepts and Calculations 17

A. The Accounting System Model 17

B. Errors and Error Models 19

C. Variance Calculations 23

D. Estimates of Error Model Parameters 27

IV. Error Models 28

A. General Error Models 28

1. Simple Error Models $\quad 28$

2. $\quad$ Regression and Calibration Models 29

3. Analysis of Variance (ANOVA) Models 30

4. Calculations Based on Ongoing Measurement Control

5. Uncorrelated Random Effects Models 34

B. Specific Measurement Problems 36

1. Holdup 36

2. Sampling 37

3. Isotope Dil ation Mass Spectrometry (IDMS) 41

4. Weights 44

5. Non-Destructive Assay 45

V. Historical ID Data and Control Limits 48

A. Model of the ID Sequence 58

B. Comparison of His orical ID Data and Variance Propagation

C. Assessing Long-Term ID Averages 53

D. The Use of Correla-ion Coefficients 54

VI. Variance Propagation Algc.rithms 56

A. A General Variance Propagation Formula $\quad 56$

B. Variance Propagation Algorithms 60

References 64

$\begin{array}{ll}\text { Appendix A } & 68\end{array}$ 
Tables

Table 2.1 Activities Related t. Inventory Difference Contrul 8

Table 5.1 Statistical Parameters 51

Table 5.2 Confidence Limits tor Different Values of $n$ 


\section{Figures}

Figure 3.1 A General Model of an Inventory Difference Calculation 19

Figure 6.1 Relative Error Model With a Random Effect E 57

Figure 6.2 Summary of a Simple Method-Oriented Variance Propagation Model 


\section{GI. Introduction}

This document provides guidance on the DOE Order 5633.3 requirements on control limit calculations. Although revision of this order was initiated while the present document was in preparation, the substance of the requirements on control limit calculation most likely will be retained in the revised order. The present document deals principally with variance propagation modelling. Chapter II addresses these requirements in terms of the need to create, maintain, and document a variance propagation model for computing control limits. It is recommended that facilities carefully allocate their effort in characterizing the uncertainties in the accounting system (with the largest contributors receiving the most attention) so that the modelling process contributes directly to controlling and improving inventory differences (IDs) as well as generating a practical method for computing useful control limits.

The rest of the document is devoted to basic technical discussion of the activities of creating and validating the variance propagation model, and developing the input data. Chapter III discusses basic variance propagation concepts. Chapter IV treat- methods for characterizing and quantifying uncertainties in the accounting system, which are the most difficult part of the modelling effort. Chapters V and VI discuss the analysis of historical inventory difference data and calculational algorithms respectively.

The first three chapters of the document are intended for a general audience; the last three chapters contain progressively more technical detail. There is already a considerable literature on statistics in nuclear material accounting (not to mention literature on applicable statistical techniques such as the analysis of variance), including a few good textbooks. The literature is strongest in statistical theory and in the treatment of situations where this theory is immediately applicable, but less strong in addressing situations where new data must be created and brought to bear on errors under poor control. Parts of chapters III, IV and V of this document provide an introductory description of the relevant statistical concepts and techniques and indicate where these are applicable or useful, but certainly do not constitute a complete treatment. The book "Statistical Methods in Nuclear Material Management" $\left[1 j^{*}\right.$ can be regarded as a primary reference; it thoroughly treats most of the statistical topics referred to in this document, the exception being the algorithms treated in chapter VI.

Two other current reports that are generally relevant to the topics discussed here are a report. on target values for measurement accuracy by New Brunswick Laboratory [2] and a report on the SAMCAT project by Argonne National Laboratory [3].

Terminology has been a source of controversy in this area. The recommendations on semantics in an NRC panel report [4] are followed in reference [1], and are largely followed here, along with some other recommendations regarding variance pri pagation modellir.

\footnotetext{
"References are identified by squa.e brackets, [ ], in the text, and are listed at the end of the document
} 
The guidance will not pre: cribe a particular variance-propagation model, but will outline a basic theory of variance propagati in and identify general characteristics that variance propagation models should have. A number of varlance-propagation computational formulations and algorithms are available, and no single approach is universally appropriate. If anything, an emphasis on a particular algorithm may tend to distort priorittes. The focus of the effort should be primarily on understanding the accounting and measurement systems. Choosing a model and "filling in the blanks" with "random errors and bias" without an understanding of what these numbers represent will seldom generate useful results. The terminology used here, and later in this document, is discussed further in Chapter II, Section B. 


\section{The Variance Propagation Requirements in DOE Order 5633.3}

The first section of this chapter interprets the wording in 5633.3 in terms of a number of activities for which the facility's MC\&A organization is responsible. The second section cilscusses the goals of these activities and addresses problems that have attended variance propagation efforts in the past. The final section discusses control limit calculations. Technical aspects of all these discussions are amplified in subsequent chapters.

\section{A. The Scope of the Requirement.}

The following three passages in DOE Order 5633.3 cover the basic requirement for control limit calculation and variance propagation:

"Control Lirnits - The established values beyond which any variation, in this case inventory difference, is ccnsidered to indicate the presence of an assignable cause. Control limits established at the $95 \%$ confidence level are called warning limits. Those established at the $99 \%$ confidence level are called alarm limits." (Definitions) "Each facility shall have a documented program for evaluating all SNM inventory differences, inclucling those involving missing items.... Procedures shall be provided for establishing control limits and requiring investigation when those limits are exceeded. Assessments of inventory differences shall include statistical tests (e.g., tests of trencls and biases), and shall be applied, as appropriate, to both total inventory difference and actual inventory difference on an individual and cumulative basis." (II.6.b.1)

"The procedures for establ:shing control limits shall be based on variance propagation or any other statistically valid technique. Historical inventory difference data shall be evaluated for comparison with statistically based limits, where applicable. Where the propagated or otherwise statistically based methods do not yield control limits consistent with historical data, efforts shall be made to resolve and reduce the difference to as low a level as possible."(II.6.b.2)

In addition, the following passages provide the requirements for measurement control programs as they relate to variance propagation:

"Each facility shall develop and implement a documented measurement control procedure for each measurement method used for accountability" (II.4.e.2)

"Each facility shall have a documented program for the statistical evaluation of measurement data for determining control limits, calibration limits, precision and accuracy levels for each $\mathrm{m}$ asurement system used for accountability. The objective is to assure the quality of me:surement and measurement control data arid to provide 
estimates of uncertainty on inventory and inventory control statements. The program, at a minimum, shall contain the following elements:

(a) A valid statistical technique to determine the total random and bias generated for each measurement system of sampling/measurement system, control limits, rejection limits, and outlier criteria; .. " (II.4.e.3)

This document is most directly concerned with the paragraphs II.6.b.1 and II.6.b.2 above. Compliance with those paragraphs entalls the establishment of a variance propagation function whose basic concern is the calculation of control limits "based on variance propagation or any other statistically valid technique". While the Order states that all inventory differences must be evaluated, clearly the use of variance propagation is not applicable to material balance areas involving solely item accounting. Furthermore, in accordance with the graded safeguards approach, the highest priority in carrying out variance propagation modelling (or other equivalent types of modelling) should be given to Category I MBAs.

This document will focus on modelling using variance propagation techniques; a large part of what follows will be devoted to the question of what constitutes an appropriate variance propagation model, how to create one, and how to go about deriving limits on the basis of the results. The words "any other statistically valid technique" are assumed to refer to simulation (or "Monte Carlo") methods. These methods do not differ greatly from variance propagation modelling from the point of view of this guidance, in that the basic results are the same (an ID variance or standard deviation) and the basic input data are the same (a characterization of the uncertainties in the accounting system). The two methods give comparable results when used with the same assumptions; if anything, simulation studies will require more data and more computational effort. Simulation techniques are applicable when the interaction between random quantities are too complex to be represented by the simple error models presented in chapter III.

On the other hand the term "any other statistically valid technique" is not meant to include procedures involving historical ID data alone. Control limits based solely on historical data cannot react quickly enough to changes in the process, to changes in throughputs, or to changes in measurement systems. However, as indicated in paragraph 11.6.b.2, quoted above, historical data must still play an important role $\mathrm{n}$ validating the variance-propagation model, as they may play a large role in what the control limits are.

As noted above, compliance with the variance-propagation requirements (paragraphs 11.6.b.1 and II.6.b.2) is satisfied by the establishment of a function (that would appropriately report to the MC\&A manager) whose basic responsibility is the computation of control limits. This activity should be closely linked with other MC\&A. activities, as described below. The function must be able to draw on statistical expertise, but also on persons intimately familiar with the accounting system, measurement systems, and the process. This function should be responsible for four types of activities:

Model Creation 
A vartance propagation model (or simulation model) consists of calculational algorithms along with associated input data. The model should have the following characteristics and capabilities:

- It should be capable of computing the variance of the ID; it is also useful to be able to compute the covariance between IDs.

- The ID standard deviations should be used to compute control limits; these limits may incorporate information on long-term ID means and previous IDs (see Section D).

- The output of the model should provide a breakdown of the computed variance which is detailed enough to allow an analysis of the extent to which various sources of uncertainty contribute to the total variance. One should also be able to determine which flow or inventory quantities are associated with the major variance terms.

- The model should be capable of reflecting current facility conditions in terms of process quantities and flowsheets.

- The model should reflect the sources of uncertainty in the accounting system and link them appropriately to inventory and flow quantities.

- The model should be flexible enough to accurately model the effects which contribute most strongly to the behavior of the inventory difference. In particular, it should be capable of modelling the correlations that exist among accounting values in a realistic manner, so that if a particular effect applies to a set of item values in reality, the same will be true in the model.

The sources of input data for the model are current values for flow and inventory quantities and quantified estimates of uncertainties (standard deviations) based on measurement control data, data from material tracking systems (a term that will be used to describe generally the type of sub. MBA data generated by process monitoring and NRTA systems), from ad hoc experiments and from expert judgement.

The model need not be more detailed than is necessary; for example, it need not contain the individual values of every item in the material balance if a stratum-level description (e.g, number of items in the stratum, average weight, average enrichment) will yield essentially the same result. Calculated ID standard deviations are not going to be accurate to a few percent in any case. In some cases, a fairly simple spreadsheet-type calculation may be quite adequate, provided the major contributors are modelled carefully.

Model Validation

The model should be validated by a comparison between historical inventory differences and the standard deviations calculated by the model under the conditions (flows, inventories, and so on) that existed during the historical periods. A situation in which computed variances significantly exceed historical values indicates that some variances have been overestimated, or that something is fundamentally wrong with the mudel. The solution can usually be found by examining the one or two largest terms in the variance sum. A situation in which historical variances exceed computed 
values generally indicates that some important effect has been omitted. Sometimes the serial correlation of the historical ID series can indicate whether the ID is dominated by uncertainties in flows or in inventories.

The major variance terms in the model should be reviewed by knowledgeable process and accounting staff to see whether these causes correspond with expectation and the actual functioning of the system. This interaction is a necessity if the model is to have a chance of reflecting reality because of the complexity of accounting systems and processing operations.

\section{Model Application, Maintenance and Improvement}

The model is run as appropriate to generate ID standard deviations and control limits. The model should be maint?ined by updating the input data to reflect current values for flows and inventories, and current estimates of error standard deviations. Changes in the accounting system, the measurement systems, or the process should be reflected in the model. Situations in which dominant terms in the variance propagation sum are not supported by quantitative results (i.e., are based on estimates or judgement) represent areas where action is needed either to eliminate or control and quantify the source of uncertainty. This process of identifying and quantifying the major sources of uncertainty in accounting systems is a major focus of the effort that should be expended in support of variance-propagation.

\section{Model Documentation}

Without an adequate level of documentation, it is difficult to maintain an understanding of what is occurring inside the model. This makes it difficult to review, and increases the likelihood that model and reality will diverge. Documentation serves the purpose of ensuring corporate memory as it does in any important program.

Documentation describing the model need not be voluminous, but should describe:

- how accounting system quantities are represented in the model;

- which sources of uncertainty are incorporated into the error models;

- what accounting system adjustments are incorporated;

- the error models;

- the source of each estimate of a standard deviation, and how it was computed.

The model itself should be able to generate the set of uncertainty estimates and (aggregate) accounting values used by the current calculation.

The three functions of model creation, validation, and maintenance are discussed in more detail in the remainder of this document.

\section{B. An Overview}

The nperational and techni :al approach to variance propagation modelling should address a number of problems that have existed historically in the calculation of variance propagation at large complex facilities: 
- Control limits calculated by variance-propagation methods have tended to be smaller (in other words, narrower) than those derived from historical ID data, raising the concern that variance propagated limits would create too many false alarms.

- The effort required to identify and model all sources of uncertainty in a real accounting system appears to be prohibitive.

- Some sources of uncertainty in accounting systems are impossible to identify or very difficult to quantify.

To some extent these issues can be addressed by properly identifying the goals of the variance propagation modelling function, and by allocating effort in the most cost-effective manner to achieve those goals. The modelling function is used to generate useful control limits, to improve the understanding of the behavior of the accounting system, and to control and improve ID performance. It should be viewed as one of a number of related activities described in Table 2.1, all aimed at these objectives. When taken in isolation and carried out without the support of the other activities shown in this table, variance propagation modelling will probably not be useful or successful. As part of a larger program, variance propagation can help identify problem areas and set priorities for upgrades ats well as supporting coritrol limit calculations.

It is impractical to try to generate a statistical model that accurately incorporates all of the sources of uncertainty that exist in a real accounting system. The problem is, as noted above, that the task of gathering enough data on all these effects requires a great deal of effort. However, a perfect model is not necessary in terms of the goals described above. Therefore, it is recommended that in order to expedite compliance with the order, rather than attempting from the start to completely and accurately characterize all elements of the accounting system, a reasonably sound model be constructed which may incorporate estimates (expert judgement) on the size of some effects for which data are not available. This forms the basis for the following cycle of analysis and improvement: 


\section{Activities Related to Inventory Difference Control}

\begin{tabular}{|ll|}
\hline \multicolumn{1}{|c|}{ Activity } & \multicolumn{1}{c|}{ Purpose } \\
Material control programs that generate data on & Control measurement processes \\
measurement systems & \\
Analysis of material control data & $\begin{array}{l}\text { Characterize measurement uncertainty } \\
\text { quantitatively }\end{array}$ \\
Ad hoc experiments to generate data on specific & $\begin{array}{l}\text { Provide information on sources of variability } \\
\text { not captured by measurement control programs }\end{array}$ \\
Analysis of ID data & $\begin{array}{l}\text { Characterize aggregate behavior of the } \\
\text { uncertainties in the accounting system }\end{array}$ \\
Analysis of material tracking system data & $\begin{array}{l}\text { Detect blunders or short-term losses; characterize } \\
\text { holdup, bias and other sources of variability }\end{array}$ \\
Variance propagation modelling & $\begin{array}{l}\text { Determine control limits, identify major causes } \\
\text { of ID behavior. }\end{array}$
\end{tabular}

Table 2.1

- The model is compared with historical data, and discussed with knowledgeable staff as discussed above. The model should be run under the appropriate historical conditions, and the historical IDs should be compared with the standard deviations predicted by the model (after appropriate adjustments are made to eliminate spurious effects).

- If the model's predictions are inconsistent with the historical data, or turn out to be flawed in some other way, the model must be corrected. If necessary, expert judgement may be used :o reassess uncertainties in the accounting system to bring the model to the point where consistency is achieved. This may mean, for example, 
adding a term for the variability of holdup in a specific location, or changing a measurement standard deviation to reflect uncertainty due to matrix effects. While this procedure may not appear scientifically ideal, the resulting model does represent the best understanding at a given point in time regarding how the accounting system is working, yields reasonable values for control limits, and forces a consideration of where the model needs to be improved. Demanding this consistency is not the same in effect as simply using historical IDs to generate control limits, principally because past and present conditions are seldom identical.

- Control limits are calculated using the model.

- Terms in the model which provide large contributions to the total variance, but which do not have a sound experimental basis are given high priority for further data gathering and investigation. Note that where such a situation exists, a significant portion of ID behavior is being generated by an uncontrolled phenomenon, so that such investigations are warranted in support of the general goal of ID control, regardless of the requirement for variance propagation. The needed data may have to be generated by ad hoc experiments or may be available from other sources, or it may be possible to eliminate the source of uncertainty altogether.

- The model is maintained by updating its input values and checking its predictions against actual IDs and other data (for example, data from material tracking systems).

This development should take advantage of the fact that generally a very small number of terms contribute significantly to the total computed ID variance. These terms need to be modelled with more care than those which are less significant. Studies aimed at estimating error standard deviations are prioritized on the basis of a fairly obvious cost-benefit principle: studies requiring relatively little effort involving uncertainties which contribute heavily to the total ID variance are done first. Sometimes it is not obvious which sources of uncertainty are most important, and some data-gathering must be done to identify the important effects before effort is expended on unimportant phenomena.

The following sections and chapters describe the modelling process in greater detail. The technical approach suggested for variance propagation modelling may be described as follows:

- All "benign" sources of uncertainty or variability which are known are characterized or estimated in the model, including all sources of measurement error and holdup variability, excluding only those abnormal situations we wish to detect: process upsets, spills, and material lost from the accounting system. The sources of data for such modelling are measurement control programs, ad hoc experiments, data from material tracking systems and, as mentioned above, expert judgement as necessary.

- Because it is not always possible to know or anticipate all the sources of uncertainty, there will necessarily be some unknown effects which will not be modelled. The 
most important of these effects usually take the form of biases which manifest thernselves as long-term gain or loss trends and/or shipper-receiver dilferences. For example, the existence of an unsuspected impurity in a product stream might generate a bias in the output measurement. It seems appropriate to treat the problem of long-term trends as a separate problem, and the Order specifies that tests be made on cumulative IDs. Tests for individual IDs can be centered around an observed long-term mean (as suggested in recent DOE guidance; see [5]). Significant long-term gain or loss trends should be investigated so that the source(s) of the bias can be eliminated.

- A particular preferred form of variance propagation model will not be identified in this document. Abstract models are detailed in the literature and computational models accompany variance propagation software. (One which is generally cited assumes that each accountancy value is a product of up to three values each of which has a relative "random error" and "bias"). No single one of these model forms can be singled out as universally appropriate; a number of different approaches are discussed below. As noted in the introduction, it is important to choose or construct the model so that it fits the characteristics of the uncertainty data, rather than trying to force the data into a particular format.

While the approach outlined here is intended to make variance propagation modelling a more practical exercise, is does not eliminate the effort needed to pursue and quantify the major sources of uncertainty in the material balance.

\section{Modelling Real Accounting Systems}

Many sources of variability affect a real inventory difference; not all of these should be modelled in the variance calculation. Because control limits will be based on the variance generated by the model, any source of ID behavior excluded from the model will tend to cause alarms, while effects included in the model generally will not. Thus the variance propagation model should portray "acceptable" or "normal" or "benign" operating conditions. Effects or causes that should trigger an investigation should be excluded. Obviously, the theft of material is one such cause. The list below indicates general causes of inventory differences and their relationship the variance propagation model.

- Measurement error: all types of measurement error effects should be modelled.

- Holdup variability: the 'rariability of unmeasured holdup should be modelled.

- Uncertainties in estimate : or factors: these should be estimated as part of the model.

- Sampling error: sampling errors should be modelled, either separately or in combination with measurement error.

- Non-routine events impacting the accountability system: spills, unusual holdup problems, and other unpredictable process conditions that affect thie measurement systems cannot be modelled; it is a legitimate function of the accounting system to alert the facility of such conditions 
- Diversion and theft: excluded from the model.

- ID variability introduced by adjustments and other accounting effects: the ID calculated for the purposes of variance propagation should be consistent with the accounting system model used for the variance propagation calculation; this may mean excluding some prior-period adjustments for the ID.

- Clerical mistakes and other "human errors": these effects are difficult to model; attempts should be made to minimize them, and to incorporate the remaining irreducible ("human error") variability into the model, using existing techniques (see [6] and [7]).

- Unknown biases and unknown streams: obviously if they are unknown they will not be modelled; they will generally show up as long-term gain or loss trends which must be investigated.

The remainder of this section discusses some of these categories in more detail.

Holdup

Holdup is defined as "the amount of nuclear material remaining in process equipment and facilities after the in-process material, stored materials, and product have been removed". Material held up in process lines or vessels is obviously part of the inventory, but it is often difficult to measure. Various approaches to the holdup problem are: measure it using NDA, estimate it by visual inspection, estimate it as a factor, clean it out, and model it statistically. In the first two cases, it is modelled as a measurement process. It is intended that the terms for holdup variability included in the variance propagation model be specific to pieces of equiprnent or locations within a facility rather than becoming a catch-all for unexplained ID variability. Holdup is discussed as a topic in chapter IV.

Uncertainties in Estimates or Factors.

Not all accountancy quantities are measured values. Most accounting systems contain "factors" or "stream average values" or "plug values" which are either fixed estimates of nuclear material quantities, or fixed values from which nuclear material quantities are calculated.. Holdup may be estimated as a fixed quantity of material that is associated with a particular piece of equipment or it may be estimated in terms of a fixed increment attributable to each operation or unit of throughput (e.g. 2 grams of material are assumed to accumulate on a filter with every batch of material processed). If a fixed value for the holdup is used and the actual holdup varies, this variation will contribute directly to the ID Factors are also used in place of measured values for quantities such as enrichment or purity which are well controlled. In any of these circumstances, the difference between the factor and the true value of the parameter can be regarded as a random effect or a combination of random effects and treated like a measurement error. This means periodically making a measurement of the parameter as would be required in a measurement control program, and modelling the error based on the results. 
Accounting System Effects

Accounting systems contribute to ID behavior through such mechanisms as prior period adjustments. This is an addition or subtraction made to an account in the present accounting period due to a revision of an accounting value in the previous period, perhaps because a better (final) value has come in for one or more items to replace an initial (temporary) value. The account appropriately credits the MBA with the difference between the temporary value and the final value. This type of process has the effect of correcting the cumulative inventory difference while possibly introducing some extra variability into the ID sequence.

The accounting system model for the variance propagation should be consistent with the actual calculation that generates the ID that is compared to the computed control limits. If that ID is based on preliminary values, the accounting system model and the error models should reflect the uncertainty in the preliminary values. If prior period adjustments do not involve items which are not in the current material balance, the adjustments are entirely spurious; the best option is to eliminate them from the ID which is to be evaluated.

Sampling

All destructive analyses and some NDA techniques require a sample to he taken which is representative of the item or batch to be measured; the measurement of the item cannot be more accurate or precise than the sampling process regardless of the quality of the measurement itself. Sampling error may be considered to be a random effect in which the error is the difference in the parameter of interest (usually concentration in liquid sampling) in the sample and the average value of the parameter in the item sampled. Obviously where sampling errors are significant they should not be ignored, although it is often convenient to model the errors due to sampling and analysis together instead of treating them as separate effects.

\section{"Human Error"}

Human error can take the form of clerical mistakes (such as transcription errors) or it can take the form of a failure to follow procedures, which in turn generates bad accountancy values (e.g. the technician fails to turn on tank agitators before taking a sample). Because of the unpredictable nature of these errors and the wide range of possibilities involved is not clear that a useful model of these types of error could be implemented, so it is not recommended that such models be attempted.

Automated systems are gradually replacing most manual systems (although some systems still contain handwritten log books) and process monitoring and material tracking systems should enable facilities, in some situations, to catch large blunders before they enter into accounting records. It appears more reasonable to attempt to reduce these types of errors to low levels than to try to model them.

\section{Measurement Uncertainty}

Models of measurement $e$ ror can be constructed on the basis of mathematical or physical theory, analysis of measurement control data, ad hoc experiments, or expert judgement. 
Unfortunately, the types of effects which are easiest to characterize are usually the least important in terms of contributing to ID behavior. In particular, the uncorrelated random effects which characterize the variability observed when repeatedly measuring the same item tend to be inconsequential, while the more highly correlated, long-term effects are both more difficult to estimate and more important numerically.

Most data on measurement errors from destructive or NDA systems should come from measurement control programs. Current orders require measurement control programs for all measurements associated with accountancy. Each measurement technique should have associated with it a program of measurement control which not only insures the proper functioning of the measurement system but provides data that can be used to build error models. Such programs generally consist of periodic measurements of "standar'd" items or samples of "known" value. In practice, a measurement control program may capture almost all or very little of the behavior of the measurement error.

For example, a non-destructive assay instrument such as a gamma-ray can scanner measures the nuclear material content of solids in cans or drums by scanning the item from top to bottom while making a transmission-corrected gamma-ray measurement. A low-level measurement control program for a can scanner might consist of counting a foil on a daily or weekly basis. This will demonstrate that the instrument is functioning, and may provide some information about errors associated with electronic drift. A second-level measurement control program would involve the fabrication of one or more "standards" which contain material like that actually measured, and require that these standards be measured periodically. Analysis of the variability of measurements made on such standards will give a better picture of measurement error than that obtained in the first case, as it will incorporate problems due to background. This standard may not have the same attenuation properties as process materials, however, and changes or variability in this characteristic of the item will affect the NDA values. A third-level measurement control program would involve (in addition to the meisurements of standards) periodic remeasurements of actual process material by other (better) techniques. The paired comparison of the can scanner values and the more accurate values will show if changes in the matrix or geometric parameters of process material are changing the error parameters of the can scanner.

Generally speaking, the reasons that a measurement control program might fail to provide adequate data are:

- The measurement control sample may not be "blind" to the operator of the instrument or the analyst. in that he may know that the sample is a control sample, and may know what the value of the sample is. Thus he will treat the measurement differently than he would otherwise.

- Differences in composition or other physical characteristics ("matrix effects") between the process material and the standards affect the measurement results.

- The standards themselves may not have well-characterized values. 
Obviously, some measurement situations will have these problems to a much greater degree than others. In general, the pure materials which constitute major flows lack the interfering impurities and are therefore measurable in well-controlled systems. Waste streams are difficult to measure because their composition varies from item to item.

Measurement systems which are under poor control will be difficult to model; but it should be noted that these situations represent problems for the material accounting system as a whole, and have to be addressed whether variance propagation is being undertaken or not. A number of possibilities exist for bringing measurement systems under better control:

- Where matrix effects cause problems, a second (controlled) technique may be used to measure a small subsample of the poorly controlled one or items may be stratified and additional standards created to minimize errors.

- Matrix effects may be controlled by creating new standards more closely reflecting process materials. Sometimes this can be done rather easily by segregating items with differing characteristics and using separate calibration curves for the batches.

- Where scrap materials are involved, recovery values may be used to estimate measurement uncertainty.

- In some circumstances, where batches are tracked through a process or where balances can be drawn around a process step, this data can be used to estimate the uncertainty of a measurement.

- Standards whose value! may have changed (e.g., weight standards corroding or being damaged or chemical standards evaporating) may be periodically remeasured. In some cases this type of problem must simply be eliminated by means of adequate administrative controls.

- Specific ad hoc experiments may be constructed to estimate the size of some effects.

As has been noted above, generally speaking measurement errors in reality may depend on a number of factors that we can identify (technician, instrument, etc.) and perhaps many that we cannot. If is feasible to identify and quantify these factors then a detailed model of the measurement error an be constructed. Often this is not the case. As is suggested in the next chapter, under circumstances in which the measurement is under reasonable control it is adequate to use a model which consists of:

- an uncorrelated effect that can be estimated from measurement control data;

- an effect due to bias-correction which is correlated over all measurements to which a given correction applies, which is also derivabie from measurement control data;

- an effect associated with the uncertainty in the standard(s); and

- an effect expressing the residual correlated error due to standard-process material differences. 


\section{Computing Control Limits}

A number of variants on the usual control limit calculation are suggested as options for computing control limits, depending on what types of correlation are observed in the behavior of the inventory difference sequence. The general form of the control limits for an individual ID is

$$
A+B \pm C D \sigma_{I D}
$$

where $A$ is a long-term ID mean, $B$ and $C$ are associated with correlations between individual IDs, D is 1.96 or 2.576 for warning and alarm limits respectively, and $\sigma_{I D}$ is the standard deviation of the ID, as calculated from the variance propagation model. The choice of whether or not to use the $A, B$, and $C$ terms is left to the judgement of the facility, provided that whatever form is chosen is used consistently.

1. Long Term Trends

Long-term gain or loss trends are usually associated with uncorrected biases in flow measurements (the other alternatives - aside from theft - being a steadily changing value of holdup or an unmeasured stream). These blases are probably uncorrected because they have not been identified, and no account has been taken for these phenomena in the variance propagation model. It is therefore reasonable to try to treat the problem of determining the significance of long-term gains and losses as an issue distinct from the issue of determining the significance of an individual ID. It is also reasonable that the response 'o a significant single ID be different than the responses to the detection of long-term trend. In fact DOE Order 5633.3 states that tests should be made both on individual and cumulative (long-term) IDs. Therefore in the above limit calculation for an individual ID, there is a term " $A$ " which in effect removes the long-term trend from the determination of significance of the individual ID.

The long-term mean term ' $A$ " is just the average ID over the last $N$ material balance periods, where $N$ must be chosen on pracical grounds. This term $A$ should only be used if the trend is statistically significant; otherwise $A$ is taken to be zero. This question is taken up in chapter $V$.

\section{Correlations Between Successive IDs}

The fact that the ending inventory of one material balance period is the same as the beginning inventory of the following ID, and enters with the opposite sign, creates a negative correlation between successive IDs. As discussed in chapter $\mathrm{V}$, this correlation produces a correlation coefficient that can range from zero to approximately $-1 / 2$ under reasonable assumptions. The $-1 / 2$ value occurs when essentially all of the uncertainty in the ID is associated with the calculation of the inventory quantities, and these inventory-related effects are uncorrelated from balance period to balance period. The negative correlation manifests itself in behavior in which a large positive ID tends to be followed by a negative ID and vice versa. In material balance areas in which the correlation is close to $-1 / 2$, it is reasonable to take this into account; for one thing the limits derived 
in this manner are slightly narrower. Use of the $B$ and $C$ terms in the limit calculation provide a compartson of the current ID with its expected value given the prevlous ID, rather than with its unconditional expected value.

The terms $B$ and $C$ in the equation above are computed as follows

$$
\begin{aligned}
& B=\rho X_{n-1} \\
& C=\sqrt{1-\rho^{2}}
\end{aligned}
$$

where $X_{n-1}$ is the previous inventory difference, and $\rho$ is the correlation coefficient. Chapter $V$ discusses the estimation of $\rho$ from historical ID data and from the vartance propagation model. Clearly if $\rho$ is close to zero, it makes very little difference whether it is included or not, and small values of $r$ will not show up as statistically significant in the historical ID. However, when $p$ is $-1 / 2$, the factor $C$ narrows the limits by about $12 \%$, and the factor $B$ can shift the limits considerably subsequent to a large ID. Where both the historical data and the variance propagation model indicate that $\rho$ is large (close to $-1 / 2$ ) the factors $B$ and $C$ can be used. 


\section{Basic Variance Propagation Concepts and Calculations.}

This chapter provides an introduction to the concepts and definitions associated with variance propagation modelling and the calculation of control limits. A more general treatment of variance propagation mathematics and algorthms is given in chapter VI. Conceptually, a variance propagation model is composed of information regarding the structure of the accounting system (how measured values are combined together to compute IDs) and information regarding the uncertaintles that relate to the basic data that go into this structure.

\section{A. The Accounting System Model}

Varlance propagation calculations must incorporate data indicating the amounts of materials in flows and inventories, and must tie this information to data on measurement methods. Figure 3.1 Illustrates the hierarchy of clata and calculations which generates inventory differences.

The fundamental data on which accounting system information and inventory differences are based are measured or estimated values of physical parameters such as weight, concentration, and enrichment. For lack of a better term, we will call these values the observed values of the physical parameters, as distinguished from the true values.

Entries are made into the accountability system for nuclear material quantities in discrete units (cylinders, tanks, cans, etc., containing nuclear material) that will be called item values. Item values are calculated on the basis of observed values based on a well-defined Item function for that item. Generally speaking, the items are grouped into sets of items or strata, and considered together because their physical characteristics are similar, and because their nuclear material content is calculated in the same manner. By definition. therefore, items within strata all use the same item function. A single tem may constitute its own stratum.

\section{Exampleз 3.1}

(a) Ten- or twelve-ton $\mathrm{UF}_{6}$ cylinders are the principle feed stream to an enrichment or fabrication facility. Cylinders of a particular size at a particular enrichment (say normal) constitute one stratum. The U-235 content of a UF 6 cylinder is calculated using the observed values of net weight, enrichment, and purity (grams $\mathrm{U} /$ gram). The item function defining the item value is given by the product:

$$
\text { Cylinder U-235 }=(\text { Net Weight })(\text { Purity })(\text { Enrichment })
$$

(b) The content of alumina traps is removed from the trap and measured directly by NDA; low-content cans of trap material may be measured by a gamma method while high-content cans would be measured by a neutron device. The two sets of 
values would constitute two separate strata. The item function for each stratum is simply

$$
\mathrm{U}-235 \text { content of } \mathrm{ttem}=(\mathrm{NDA} \text { value })
$$

While the item function is the same for the two methods, error models for the two methods may differ.

The item values of all items within a stratum are summed to arrive at a stratum value; stratum values are summed to compute a value of one of the four ID components: beginning inventory, ending inventory, additions, or removals. These components are appropriately added and subtracted to compute the ID

A specific accounting system can be modelled in more or less detall, depending on the use made of the concept of observed values. A concentration value is generated by the lab based on other measured values such as lab weights; in theory one could use these more fundamental observations in the model. On the other hand, one could irnagine using item values or even more aggregate data as the observed values. The appropriate level of detail is generally fairly obvious; it is the most aggregate level at which direct information is available on the error characteristics of the observation. We have no direct information on the uncertainty of the plutonium contents of a tank of solution, but measurement control programs and tank calibration data relate to volume and concentration measurements separately.

The term static inventory is used to describe items which are on both beginning and ending inventories at the same value; in sther words, inventory to which nothing has happened and which was not remeasured during the mtterial balance period. These items effectively drop out of the ID calculation, and so must be treated carefully in variance propagation calculations. 


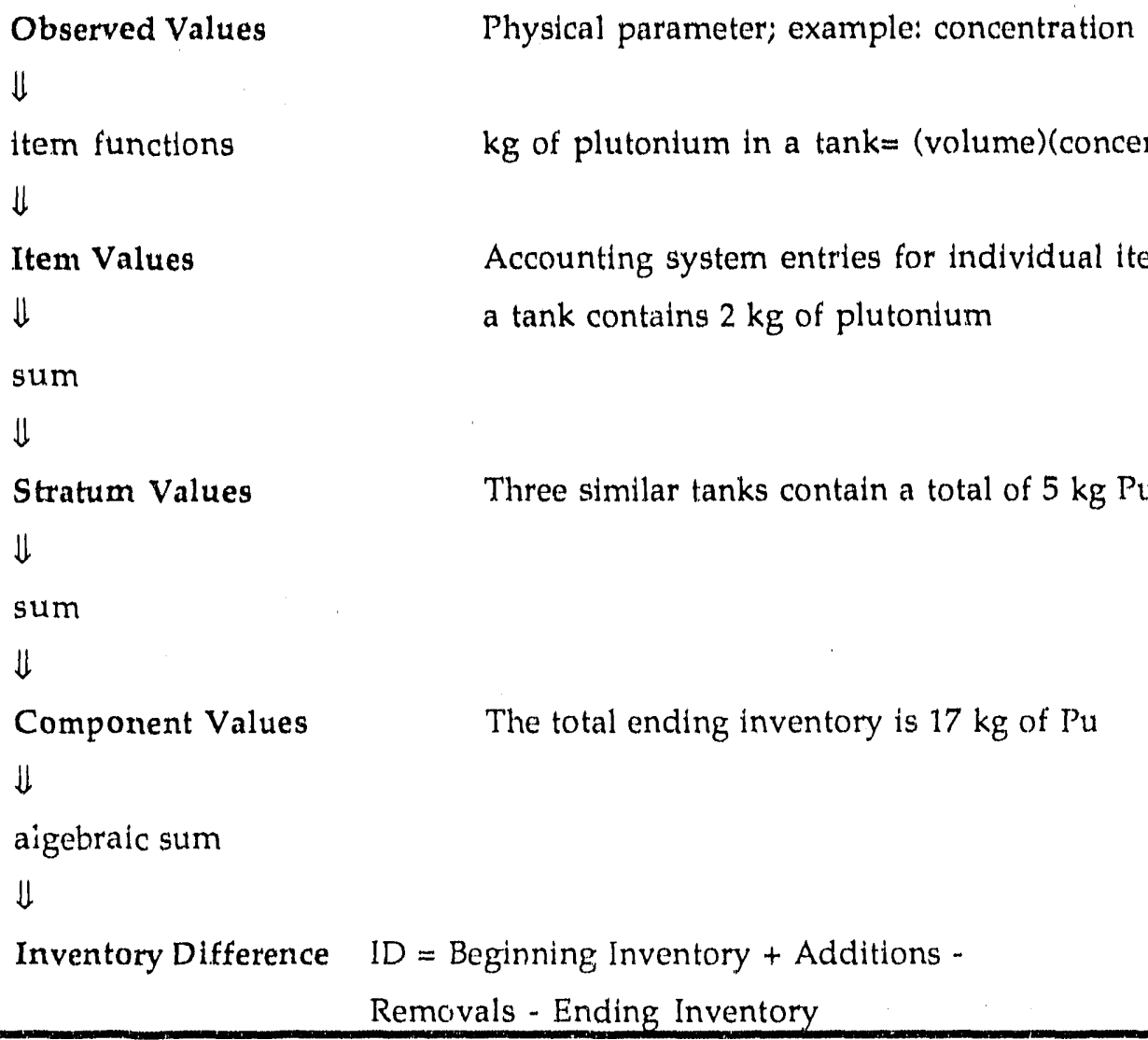

Figure 3.1

B. Errors and Error Models

Generally speaking, because of measurement error (and other uncertainties) the observed values and true values of a physical parameter will not be identical. The difference between the two values will be referred to as an error. [This usage of the word "error" is common although it is incorrect to associate these types of errors with the concept of mistakes.]

Variance propagation calculations begin with a mathematical characterization of accounting errors sometimes known as measurement error models or simply error models. An error model is an equation relating the observed value, the true value, and one or more types of errors. In simplest form these equations appear as follows:

$$
m=\mu(\uparrow+\varepsilon) \quad \text { (relative error model) }
$$


or

$$
\mathrm{m}=\mu+\varepsilon \quad \text { (absolute error model). }
$$

Here $m$ is the observed value, $\mu$ is the true value and $\varepsilon$ is the error (relative or absolute, as appropriate).

The first equation above represents a relative error model, because the error is expressed in relative (or dimensionless or percent) terms. The second model is called and additive or absolute error model, because the error is expressed in the same units as the observed value. Additive error models are used in the situation where the value of the paramete: involved does not affect the magnitude of the error, whereas a relative error model is used where the magnitude of the error tends to be proportional to the parameter.

The error terms " $\varepsilon$ " in the models above are regarded as the results or realizations of random variables:: numbers subject to statistical variability or uncertainty. For the purposes of this document, random variables are primarily characterized by their mean (or average value), their standard deviation (describing, loosely speaking, the average amount by which the random variable will differ from its mean), and the degree of correlation between random variables. Errors which have distinct and unrelated physical causes are modelled as the combination of uncorrelated random variables. The variance of a random variable is the square of its standard deviation. It is more convenient to compute with variances than with standard deviations, but variances are expressed in difficult-to-interpret units (such as kilograms-squared), so calculation are carried out with variancess and the square root is taken to yield a standard deviation as an answer.

\section{Examples 3.2}

(a) A pressure gauge display is divided into fifty divisions, which represents a pressure range from 5 to $10 \mathrm{psi}$. It is assumed that the gauge can be read to "about 1 division". In the absence of more precise data, the one division can be equated with about 2 standard deviations. Since a division always represents 0.1 psi the reading error could be modelled as an additive error with $s$ (the standard deviation $)=0.05$ psi.

(b) An electronic balance rounds its results to the nearest gram. Again, the error is of the same magnitude regardless of the weight involved, so the rounding error is modelled as additive. The standard deviation of a rounding error can be calculated as approximately 0.29 times the size of the digit rounded, in this case 0.29 grams.

(c) Many chemical and NDA assay techniques have uncertainties quoted in percent, as the errors are assumed to increase with the quantity measured. Usually an 
"uncertainty" quoted as " \pm " represents two standard deviations; sometimes (for example with documentation distributed with standards) uncertainties are quoted conservatively at three standard deviations.

It should be noted that the distinction between additive and relative error models may or may not be important in practice. In example 3.2(b) the rounding error clearly behaves as an additive effect with a standard deviation of 0.29 grams. However, if the objects which are being weighed on the scale are always in the range of $2-2.5$ kilograms, there would be no problem in modelling the situation in terms of a relative error of approximately $0.29 / 2000 \approx .000015$. However, if the same scale were then used to measure different items, and the same relative value was applied when items of 100 grams were being weighed, the standard deviation of the error would be too low by a factor of 20 .

Random variables representing the errors in error models are usually assumed to have mean zero and are assumed to be independently distributed; in addition they are often assumed to be uncorrelated, which has advantages in terms of calculation. Situations in which this is not the case can be addressed by adding constants and extra error terms to the model, which apply to more than one measurement or observed value.

\section{Example 3.3}

An NDA instrument is used to measure four items per day; it is recalibrated daily, using the same standard every day. Three types or categories of errors are identified: the error associated with the counting statistics of the instrument, the error associated with the calibration proceaure, and the error associated with the uncertainty in the value of the calibration siandard. The three types of error arise from different phenomena, so are treated as uncorrelated. Each calibration error applies to four measurements, and the error associated with the calibration standard apples to all measurements. The measurement model is as follows:

$$
\begin{aligned}
& \text { Day } 1 \\
& \mathrm{~m}_{1}=\mu_{1}+\varepsilon_{1}+\delta_{1}+\eta \\
& \mathrm{m}_{2}=\mu_{2}+\varepsilon_{2}+\delta_{1}+\eta \\
& \mathrm{m}_{3}=\mu_{3}+\varepsilon_{3}+\delta_{1}+\eta \\
& \mathrm{m}_{4}=\mu_{4}+\varepsilon_{4}+\delta_{1}+\eta
\end{aligned}
$$

Day 2

$$
\begin{aligned}
& m_{5}=\mu_{5}+\varepsilon_{5}+\delta_{2}+\eta \\
& m_{6}=\mu_{6}+\varepsilon_{6}+\delta_{2}+\eta \\
& m_{7}=\mu_{7}+\varepsilon_{7}+\delta_{2}+\eta
\end{aligned}
$$




$$
m_{8}=\mu_{8}+\varepsilon_{8}+\delta_{2}+\eta
$$

etc.

Here, the " $\varepsilon$ " errors represent the counting statistics errors, the " $\delta$ " errors represent the calibration errors, and " $\eta$ " represents the error in the standard. All of the $\varepsilon_{\mathrm{i}}$ are assumed to be uncorrelated but to have the same standard deviation $\sigma_{\mathrm{e}}$. Similarly $\delta_{1}$ and $\delta_{2}$ are uncorrelated but have the same standard deviation $\sigma_{\mathrm{d}}$, and the error $\eta$ has standard deviation $\sigma_{\eta}$. The $\varepsilon^{\prime}$ s, $\delta$ 's, and $\eta$ errors are all uncorrelated with one another.

In the example above, the set of errors due to counting statistics are generally referred to as "random errors"; random errors are those which arise from effects that are independent or uncorrelated from one instance of measurement or observation to the next. The term "bias" is applied here to the types of errors like the error " $\eta$ " in the above example, which affect all of the data values $m$ in the same manner. A frequent, broader, usage of this term (" bias") encompasses all deviations of the expected value of a random variable from the corresponding correct value. This broader usage thus includes intermediate circumstances exemplified by the calibration errors " $\delta$ ", which are often labeled "short term systematic" errors or "partially systematic" errors. (This latter terminology has been includet because it has become commonplace, but will not be followed here. )

Following the recommendations of a panel study [4] the three sets of errors described above will be termed random effects (e.g., a random effect due to counting statistics, a random effect due to calibration, etc.). The implication of the term "random effects" is that all eight of the counting statistics errors, for example, are modelled as the values of random variables with the same distribution (and therefore the same standard deviation); a similar situation exists for the two calibration errors. The error in the standard is regarded as a single realization of a random variable with some standard deviation (which might be estimated from measurement data obtained in the experiment creating the standard). The random effect due to counting statistics is described as uncorrelated, and the random effect due to the standard is described as totally correlated. The calibration effect is described as having a correlation extending over all measurements within a given day. This terminology is cumbersome, but it is important for the purposes of variance propagation to define the number of measurements affected by any given error. To reiterate, the term "effect" refers to a collection of (one or more) errors or random variables, each of which may appear in one or more error models, each of which has the same standard deviation (that associated with the effect).

The strict use of the term "constant bias" is confined to a constant term that has no uncertainty associated with it. In terms of mathematical models, the rules that apply to such "biases" and to "totally correlated random effects" are quite clear. When applying these terms to real measurement systems, the distinction between such a "bias" and a "correlated random effect" is very subtle and has been the source of controversy (see, for example, [8], [9], [10], and [11]). In the context of 
variance propagation, it is important to remember that something that is modelled as a strict constant bias will not contribute to the ID variance (although it may contribute to an ID mean) but a correlated random effect will. When estimating the historical variance over say, two years time, we compute the historical variance about its mean; so very-long-term "systematic" effects will show up in this mean rather than in the standard deviation, and so should be treated as strict constant biases. It may sometimes be possible to characterize uncertainties in a measurement process in a very detailed manner. For example, it is often suggested that a model of the uncertainty in a chemical analysis measurement might look something like

$$
m=\mu+\varepsilon+\eta+\delta+v
$$

where $\delta, \eta$, and $v$ represent random effects associated with operators, instruments and shifts, respectively, and $\varepsilon$ is a completely uncorrelated random effect associated with each measurement. Given enough data, these effects can be characterized using analysis-of-variance (ANOVA) techniques. Often, however, such data are not available, or cannot be used because the errorpropagation data base does not contain the information to make use of the characterization. It should be pointed out that writing down an error model does not make the real error behave according to the model. Usually the error model associated with a measurement system is much simpler than the reality it represents. The simplification is generally acceptable because we are usually interested in the statistical behavior of sums of errors, whose characteristics do not differ greatly between the simple and complex characterizations.

\section{Variance Calculations}

Since errors can be modelled as random variables, the ubserved values, which are functions of the errors through the error models shown above, can be rnodelled as the results of random variables as well, with standard deviations which depend on the item functions and the standard deviations of the observed values. Finally, stratum totals, beginning and ending inventories, and the inventory difference, can all be modeled in terms of random variables. The essence of the variance propagation in the current context is the calculation of the variance (or standard deviation) of the random variable representing the inventory difierence based on a knowledge of the standard deviations of the random variables modelling the errors.

In theory, a variance propagation calculation could be undertaken by

(a) writing down a large equation for the ID which is illustrated in figure 3.1,

(b) substituting error models such as those described in the last section for all the observed values, and

(c) finding the variance of the resulting expression by means of algebraic simplification and by repeatedly applying a number of rules for computing the 
variance of functions of random variables. This process is treated in more generality in chapter VI, and in more detail in some of the references (see [1] and [12]), but the fundamentals will be described here.

Elementary variance propagation calculations can be based on four rules:

1. The variance of a constant term is zero.

2. If $\mathrm{C}$ is a constant and $\mathrm{e}$ is a random variable, then

$$
\operatorname{Var}(C \varepsilon)=C^{2} \operatorname{Var}(\varepsilon)=C^{2} \sigma_{\varepsilon}^{2}
$$

3. If $\varepsilon_{1}$ and $\varepsilon_{2}$ are random variables, then

$$
\operatorname{Var}\left(\varepsilon_{1}+\varepsilon_{2}\right)=\operatorname{Var}\left(\varepsilon_{1}\right)+\operatorname{Var}\left(\varepsilon_{2}\right)+2 \operatorname{Cov}\left(\varepsilon_{1}, \varepsilon_{2}\right)
$$

where $\operatorname{Cov}\left(\varepsilon_{1}, \varepsilon_{2}\right)$ is the covariance of $\varepsilon_{1}$ and $\varepsilon_{2}$, and is zero if $\varepsilon_{1}$ and $\varepsilon_{2}$ are uncorrelated.

4. Errors stated as relative are small in comparison to 1, and errors stated in absolute terms are small relative to the true value of the parameter, so that second-order terms in the errors may be neglected.

\section{Example 3.4}

Following example 3.3 , the total content of 8 cans of material over two days is measured as

$$
\sum_{i=1}^{8} m_{i}=\sum_{i=1}^{8} \mu_{i}+\sum_{i=1}^{8} \varepsilon_{i}+4 \delta_{1}+4 \delta_{2}+8 \eta
$$

In computing the variance of the total content the $\mathrm{m}_{\mathrm{i}}$ terms are constants and so can be ignored, according to rule 1 . The three other terms on the right hand side of the equation are all uncorrelated by assumption so

$$
\left.\operatorname{Var}\left(\sum_{i=1}^{8} m_{i}\right)=\operatorname{Var}\left(\sum_{i=1}^{8} \varepsilon_{i}\right)+\operatorname{Var}\left(4 \delta_{1}\right)\right)+\operatorname{Var}\left(4 \delta_{2}\right)+\operatorname{Var}(8 \eta)
$$

According to rule 2 above, 


$$
\operatorname{Var}(8 \eta)=8^{2} \operatorname{Var}(\eta)=64 \sigma_{\eta}^{2}
$$

and

$$
\operatorname{Var}\left(4 \delta_{1}\right)=4^{2} \operatorname{Var}\left(\delta_{1}\right)=16 \sigma_{\delta}^{2}
$$

According to rule 3 ,

$$
\operatorname{Var}\left(\sum_{i=1}^{8} \varepsilon_{i}\right)=8 \operatorname{Var}\left(\varepsilon_{i}\right)=8 \sigma_{\varepsilon}^{2}
$$

so that the variance of the total amount of material in the stratum is $8 \sigma_{\varepsilon}{ }^{2}+32 \sigma_{\delta}{ }^{2}+$ $64 \sigma_{\eta}^{2}$

The above example illustrates the generally applicable conclusions that

(a) the variance attributable to an uncorrelated random effect is proportional to the number of items, and

(b) the variance attributable a totally correlated effect is proportional to the square of the number of items.

\section{Example 3.5}

Following example 3.1(a), the U-235 in a $U_{6}$ cylinder is calculated as a product of weight, purity, and enrichment. Suppose we have the following error models for these three measurements:

$$
\begin{array}{ll}
w=\mu_{w}\left(1+\varepsilon_{w}\right) & \begin{array}{r}
\text { (the standard deviation of the relative weight } \\
\text { error } \left.=\sigma_{w}\right)
\end{array} \\
\varepsilon=\mu_{\mathrm{e}}\left(1+\varepsilon_{\mathrm{e}}\right) & \begin{array}{r}
\text { (the standard deviation of the relative } \\
\text { enrichment error } \left.=\sigma_{\mathrm{e}}\right)
\end{array} \\
\mathrm{p}=\mu_{\mathrm{p}}\left(1+\varepsilon_{\mathrm{p}}\right) \quad & \begin{array}{l}
\text { (the standard deviation of the relative purity } \\
\text { error } \left.=\sigma_{\mathrm{p}}\right)
\end{array}
\end{array}
$$

Then the product wep is

$$
\text { wep }=\mu_{w} \mu_{e} \mu_{p}+\mu_{w} \mu_{e} \mu_{p} \varepsilon_{w}+\mu_{w} \mu_{e} \mu_{p} \varepsilon_{p}+\mu_{w} \mu_{e} \mu_{p} \varepsilon_{e}
$$

plus terms involving prolucts two or more error-values, which can be neglected according to rule 4 .

The variance of the product is then 


$$
\left(\mu_{w} \mu_{e} \mu_{p}\right)^{2}\left[\sigma_{w}^{2}+\sigma_{\varepsilon}^{2}+\sigma_{p}^{2}\right]
$$

Example 3.5 points up the fact that when the item model has a product form (which is often the case in nuclear material accountancy) and when the error models are relative, the variance component can be computed using a product of the square of the item content (i.e., $\left(m_{W} m_{e} m_{p}\right)^{2}$ ) and a sum of error variance terms.

\section{Example 3.6}

A stratum of $\mathrm{UF}_{6}$ cylinders is measured as in example 3.5, but now the error models specify for enrichment (e), purity (p), and weight (w), uncorrelated ( $\varepsilon$ ) and totally correlated $(\eta)$ random effects for each of $\mathrm{N}$ items with index $\mathrm{k}$ :

$$
\begin{aligned}
& w_{k}=\mu_{w, k}\left(1+\varepsilon_{w, k}+\eta_{w}\right) \\
& p_{k}=\mu_{p, k}\left(1+\varepsilon_{p, k}+\eta_{p}\right) \\
& e_{k}=\mu_{e, k}\left(1+\varepsilon_{e, k}+\eta_{e}\right)
\end{aligned}
$$

We wish to find the variance of the observed value of the stratum total:

$$
S=\sum_{k=1}^{N} w_{k} p_{k} e_{k}
$$

If all of the items have approximately the same enrichment, purity and net weight, so that they have approximately the same U-235 content, $x=$ wpe, then application of the rules demonstrated in the last two examples yields

$$
\operatorname{Var}(S)=N x^{2}\left[\sigma_{\varepsilon, \mathrm{e}}^{2}+\sigma_{\varepsilon, \mathrm{p}}^{2}+\sigma_{\varepsilon, \mathrm{w}}^{2}\right]+N^{2} x^{2}\left[\sigma_{\eta, \mathrm{e}}^{2}+\sigma_{\eta, \mathrm{p}}^{2}+\sigma_{\eta, \mathrm{w}}^{2}\right]
$$

The above model can be used in simple accounting situations; each component of the material balance (beginning inventory, additions, removals, ending inventory) is divided up into strata of similar items, and each item is assumed to have an item function in the form of a product, and that the errors can be characterized in relative terms. The random effects are either uncorrelated ("random errors") or correlated over the stratum ("bias"). The formula above is applied to cach stratum and the stratum values summed over all components to get an ID variance. Static inventory is not taken into account by such a calculation so the static items must be subtracted from the $\mathrm{N}$ values before the calculation is made. 
If there are any effects which are correlated between strata, such a model will not handle them properly. For example, there may be two product (ending inventory) strata in the model because the standard deviations for the enrichment measurement effects differ for different enrichments. However, the purity measurement is identical for the two strata, and the correlation of the "systematic" effect should extend to both strata (in other words it should be modelled like the " $\eta$ " error rather than the " $\delta$ " error of example 3.3). The model is too simple to cope with situations in which a measurement technique applies to more that one stratum. A more sophisticated model would be based on the effects and sum over strata, so that the expression for the contribution of the purity effect which was correlated over stratum 1 and 2 would be written

$$
\left[N_{1} x_{1}+N_{2} x_{2}\right]^{2} \sigma_{\eta, p}^{2}
$$

It is often important that correlated effects such as this be handled correctly. Chapter VI provides an example of a simple extension of this model which is much more flexible and more easily adapted to real circumstances.

\section{Estimates of Error Model Parameters}

In all of these considerations it has been assumed that the values of the variances or standard deviations associated with uncertainty effects are known. Generally this is not the case; these numbers have to be calculated on the basis of observations. The results of such calculations are called estimates of the variances or standard deviations; providing good estimates of the standard deviations in error models is a prime concern in variance propagation modelling. We will distinguish the estimate of a quantity from the real quantity by placing a hat over it:

\section{$\hat{\sigma}$ estimates $\sigma$.}

Estimates are subject to statistical uncertainty, and so it sometimes becomes necessary, for example, to discuss the standard deviation of an estimate of a standard deviation. 


\section{Error Models}

The first section of this chapter discusses a number of basic general approaches to constructing error models for use in variance propagation calculations. There is very little new here in the way of statistics, and except where otherwtse reforenced, the basic theory can be found in chapters 5,6 and 15 of reference [1]. The section attempts to demonstrate how common statistical analyses relate to crror models, and then how these error models fit into variance propagation sttuations. The rest of the chapter provides some spectific examples; these are intended to be representative rather than allinclusive.

\section{A. General Error Models}

\section{Simple Error Models}

It was suggested in chapter Il that the effort expended in characterizing a particular error effect be related to the importance of that effect; that is, the fraction it contributes to the total ID variance. Although in theory this fraction is not known until the effect is characterized, in practice one generally knows when a measurement system will account for a few percent or less of the total variance. The mathematics of variance propagation guarantee that only a gross misunderstanding of such effects will have a significant impact on the final calculated value, In such cases it is appropriate to use a simple or approximate error model; usually this takes the form:

$$
m=m+\varepsilon+\eta
$$

or

$$
m=m(1+\varepsilon+\eta)
$$

where $\varepsilon$ represents an uncorrelated effect and h represents a completely correlated effect. This is the only type of error model supported by some variance propagation algorithms.

This model can be used even for important strata if care is taken in identifying what the "random" (uncorrelated) and "bias" (correlated) effects represent, and if their standard deviations are estimated accordingly.

The random $\varepsilon$ effect is usually computed as if it represents an "item-level" effect (i.e., uncorrelated between ltems) and may be underestimated by some procedures that estimate "measurement-level" variability.(sce subsection 5 below).

The correlated ("bias") effect $\eta$ must model all the correlated effects in the measurement system. These include the uncertainty associated with standards, with calibration procedures, with bias corrections, and with uncertainties not captured by measurement control programs such as matrix effects. In lumping all these effects together, one must be aware of how the variance propagation algorithm will handle the effect; for example, such systematic terms may "cancel out" 
when the measurement contributes to terms which enter the ID with equal signs. This may or may not be approprtate.

\section{Regression and Calibration Models}

In a regression application, a function $y=f(x)$ is determined based on a set of data pairs $(x \mid, y)$ ). Regression models are used in a variety of forms and for a variety of purposes in accounting systems, the most prominent example being tank calibration. Regression models may take the form of simple lineat regression, polynomial fits, or multiple regression. Tank calibration curves may involve multiple fitted segments The error models may use an independent or cumulative structure for the errors of the predicted values $y$, and there may be one or multiple sets of data (e.g., caltbration runs). Calibration and regression problems are treated extenstvely in the literature (see [13], [14], [15], and [16]). The more sophisticated treatments for the tank calibration application, involving rolatively frequent recalibration, are generally accorded to input or output accountability tanks, while intermediate process vessels are given less frequent and less elaborate attention.

Regression models will generate estimates of the parameters of the model and their standard deviations (e.g., the slope and intercept of a line), as well as estimates of the standard deviation of the value $y(x)$ of the regression equation at any given point $x$; variance estimators may be identifled with effects in error models much as in the ANOVA case discussed below.

For example, suppose the holdup in a salt bath between cleanouts is estimated using a linear regression model, with " $x$ " grams of uranium that was processed in a bath. Because the estimators of slope $\left(\beta_{1}\right)$ and intercept $\left(\beta_{0}\right)$ are orthogonal when coordinates are chosen such that the average value of the $x_{1}$ (denoted by $\bar{x}$ ) is zero, the difference between the "true" value of the caltbration line and the calculated or estimated calibration curve may be expressed by the error model:

$$
\hat{y}(x)-y(x)=\eta_{0}+\hat{\beta}_{1}(x-\bar{x}) \eta_{1}
$$

where $\eta_{0}$ and $\eta_{1}$ have variances

$$
\operatorname{Var}\left(\eta_{0}\right)=\frac{\hat{\sigma}_{0} 2}{n}
$$

and

$$
\operatorname{Var}\left(\eta_{1}\right)=\frac{\hat{\sigma}_{0}^{2}}{\sum_{i}\left(x_{1}-\bar{x}\right)^{2}}
$$

where $\hat{\sigma}_{0}{ }^{2}$ is the estimate of the residual variance of the regression. (Sec, for example, $|1|$, p.352f; this procedure is tantamount to calculating the standard errors of the regression coefficients.) The actual value of the holdup presumably varies randomly around this regression line with a varlability 
characterized by $\hat{\sigma}_{0}{ }^{2}$, assuming that the recovery values are accurate. An error model for the uncertainty in the modelled holdup as a function of $x$ therefore is:

$$
\hat{h}(x)-h(x)=\eta_{0}+\hat{\beta}_{1}(x-\bar{x}) \eta_{1}+\varepsilon
$$

where $\varepsilon$ represents an uncorrolated effect with variance $\sigma_{0} 2$. Since the $\eta$ errors are completely correlated and do not change, the model for the error in the difference in holdup between beginning and ending inventories, taken at throughput values $x_{b}$ and $x_{c}$, for example, would be

$$
\hat{\beta}_{1}\left(x_{b}-x_{e}\right) \eta_{1}+\varepsilon_{b}-\varepsilon_{\theta}
$$

In a situation like tank calibration, the calibration curve is used in a reverse manner; the value of $y$ is given and a value of $x$ derived from the calibration curve. Again using the fact that if $\bar{x}=0, \beta_{1}$ and $\beta_{0}$ will have orthogonal estimators (and $\bar{y} \approx \beta_{0}$ ), a model for the error $\ln x$ due to the calibration would look like:

$$
\hat{x}(y)-x(y)=\hat{\beta}_{1}^{-1} \eta_{0}+\hat{\beta}_{1}^{2}(y-\bar{y}) \eta_{1}
$$

where $\eta_{1}$ and $\eta_{0}$ are as before, and represent correlated effects. (The detalled development may be found in [1], Section 15.1.1, p. 352.) This expresston does not include the error effect due to the uncertainty in $y$ itself. The topic of combining the uncertaintles from various effects in complex functions is treated in chapter VI.

The idea fllustrated by this example, which is used throughout this section, is that most statistical models involve a number of orthogonal (or independent) variance estimators, and that an error model can be constructed by assigning an effect to each of these estimators in a fairly obvious way. The value of the estimator is used to estimate the standard deviation of the effect.

\section{Analysis of Variance (ANOVA) Models}

When it is desired to characterize the behavior of a measurement system in terms of one or more identified causes of uncertanty, error models can be created on the basis of the results of ANOVA calculations. The use of ANOVA techniques is covered in many places in the literature (see $|1 \%|$; also [1], chapter 5, and [12], p.100); this section will briefly indicate the way in which they might be applied to the construction of error models. As an example, suppose that a chemical measurement is performed by a number of technicians who have qualifted for that technique. It is suspected that the results of different techniclan's measurements will be offset with respect to one another; that is, that 
there is an error effect assoctated with the techntelan. Each of 'T techntelans measures a standard $N$ times to assess this effect. The stattattcal model of the experiment is assumed to be:

$$
m_{1, t}=\mu_{1, t}+\eta_{t}+\varepsilon_{1, t}+\delta \quad i=1 \ldots N, t=1 \ldots T
$$

where the indices 1 and $t$ hidicate the 1 th measurement made by techndelan $t, \eta_{t}$ is the error associated with techndcian $t, \delta$ is an error affecting all measurements, and $\varepsilon$ represents a totally uncorrelated effect affecting each measurement independently. The resultant data

$$
\Delta_{l, t}=m_{l, t}-\mu_{i, t}=\eta_{t}+\varepsilon_{1, t}+\delta
$$

can be treated as a one-way ANOVA problem. From the values of $\mathrm{MS}_{W}$ (the "within treatment" sum of squares) and $\mathrm{MS}_{b}$ (the "between troatment" sum of squares)

(a) the standard deviation of the $\varepsilon S, \sigma_{\varepsilon}{ }^{2}$; can be estimated ( $=M S_{W}$ ),

(b) the value of $\sigma_{\eta}^{2}$ can be estimated $\left(=\left(M_{b}-M S_{w}\right) / N\right.$ ), and

(c) the hypothesis that the operator effect is zero can be tested (using the

Fustatistic $M S_{b} / M S_{W}$ ). The significance of $\delta$ can also be tested (see [1], p.784).

If such an ANOVA model is used as a basis for an error model in a variance propagation, data will need to be supplied to the computation on the effects that exist at the time; if $t 1, t 2, \ldots$ etc., measurements are performed by the technicians, the error model for the measurements that go into the variance propagation model looks like:

$$
\begin{aligned}
& m_{1}=\mu_{1}+\eta_{1}+\varepsilon_{1}+\delta \\
& m_{t 1}=\mu_{t 1}+\eta_{1}+\varepsilon_{t 1}+\delta \\
& m_{t 1+1}=\mu_{t 1+1}+\eta_{2}+\varepsilon_{t 1+1}+\delta \\
& m_{t 1+t 2}=\mu_{t 1+t^{2}}+\eta_{2}+\varepsilon_{t 1+t 2}+\delta
\end{aligned}
$$

and so on.

More complicated situations with larger numbers of effects can be constructed using higherorder ANOVA models. For example, a model with an additional offec' $v$ for an instrument effect $v$ could also be constructed: 


$$
m_{1, t}=\mu_{1, t, k}+\eta_{t}+v_{k}+\varepsilon_{1, t, k}+\delta \quad 1=1 \ldots N, k=1 \ldots K, t=1 \ldots T
$$

and analyzed in a two-factor ANOVA model.

ANOVA techniques can be used in a wide vartety of situations to estiminte crror-model parameters.

4. Calculations Based on Ongoing Measurement Control Programs and Blas Corrections.

Experience Indicates that the behavior of real measurement systems is often very complicated because of practical, essentially unquantifiable parameters or difficult to define aspects of the modelling. For example, the measurement control data may not accurately reflect the actual measurement vartances, because the "blind" standards may be better known to, and measured by, the operators than other materials, and the standards used may not properly represent the materials being measured. Further, the examination of measurement control data sequences suggests the existence of correlated effects, and of blases which which change with time (see the remarks about equation (15) below). Although it would be nice to be able to generate valid ANOVA-type models of the type described previously, it is often difficult because of the effort involved and the data required. Generally data avallable for the characterization of error models are the periodic results of standards measurements generated by an ongoing measurement control program. This section assumes that such data is representative of the measurement errors on process material; if not, one of the strategles discussed at the end of chapter III has to be aclopted.

One approach to the problem of generating an error model based on measurement control clata is to assume a very simple form for the model and use the standards data to estimate the crror standard deviations. The model often adopted is

$$
m_{i}=\mu_{i}+\varepsilon_{i}+\eta
$$

where the $E$ 's represent a random effect which is totally uncorrelated between measurements, and $h$ represents a bias or correlated random effect. This is a rcasonable approach, but one must keep in mind that it will probably be a simiplification of reality; a dominant term in the variance propagation calculation might warrant a different treatment. The variance of the $e^{\prime} s$ is estimated in the usual way as

$$
\hat{\sigma}_{\varepsilon}{ }^{2}=S^{2} ; \quad S^{2}=\left(\frac{1}{N-1}\right) \sum_{i=1}^{N}\left(\Delta_{i}-\bar{\Delta}\right)^{2}
$$




$$
\Delta_{i}=m_{1}-\mu_{0} ; \quad \bar{\Delta}=\frac{\sum \Delta_{1}}{N}
$$

where now $\Delta_{l}$ represents the difference between the measured and known value $m_{0}$ for the ith standard measurement.

The error $\eta$ is intended to apply to all the measurements $m_{1}$; it is somewhat difficult to deal with. If it is modelled as a bias it effectively disappears from the vartance propagation calculation. The alternative suggested by Jaech $([12], \mathrm{p} .89)$ is to treat it as a completely correlated random effect with an estimate of the vartance

$$
\hat{\sigma}_{\eta}^{2}=\bar{\Delta}^{2}
$$

This is the best that can be done, but it is in effect a one-degree-of freedom estimate of a variance, and as such highly uncertain. It is also not clear how well this type of model will work if the actual behavior of the measurement system differs from the simple model defined above.

The use of blas corrections alleviates these problems. The issue of making or not making blas corrections is a complicated one, subject to a number of different viewpoints. From the point of view of ID calculations and ID evaluation, it is probably fair to say that where the standards measurements generate errors representative of errors on process material, a procedure of always bias correcting is generally a sound strategy. The blas corrections might be applied to each item in the accounting system, or the aggregate correction be applied as an adjustment. The blas-corrected value is

$$
\mathrm{m}_{1}^{\prime}=\mathrm{m}_{1}-\bar{\Delta}
$$

If this bias-correction is applied, the measurement model can be thought of as looking like

$$
m_{i}^{\prime}=\mu_{i}+\varepsilon_{i}+\eta^{\prime}+\eta^{\prime \prime}
$$

where $\varepsilon$ is as before (and $\sigma_{\varepsilon}^{2}$ is computed as before), but $\eta^{\prime}$ is a correlated effect with variance

$$
\hat{\sigma}_{\eta}{ }^{2}=\frac{\hat{\sigma}_{\varepsilon}^{2}}{N}
$$

where $N$ is the number of standards measurements made, and the correlation of this effect extends over all measurements to which the bias correction applles. The term $\eta$ " represents the error in the standard; this effect is correlated over all measurements for which the standard is used to bias correct (generally the whole material balance period). It turns out that this formulation is fairly robust for the purposes of variance propagation. In other words, if bias correction is being carried out, one can use this approach even though the real underlying behavior of the measurement system is more complex than the simple model defined at the beginning of the section. This is true provided that the standards measurements are randomly interspersed with respect to the correlations (e.g, if there is a technician effect, as described in the previous section, the standards measurements cannot all be made by one technician). 
This same type of logic can be applied to the uncertainty introduced by "factors" or "stream average" quantities, which are fixed values substituted into accounting system item functions because process conditions dictate that these numbers fluctuate very little. Presumably there is some program of periodic measurement of these values to confirm the continuing validity of the factor. The measurements can be treated as the "standards measurements" in a material control program. The obvious best estimate for the factor is the mean of the measured values.

\section{Uncorrelated Random Effects Models}

In any of the types of models mentioned previously, the uncorrelated effect is essentially estimated on the basis of the residual mean sum of squares. There are a few simple ways to estimate the standard deviati: of uncorrelated effects which are generally mentioned and will be repeated here. Estimating the standard deviation associated with the uncorrelated component of a measurement error is generally not a difficult problem in theory. In practice, it is important to keep in mind that "uncorrelated" in a variance propagation model generally means uncorrelated from item to item, rather than measurement to measurement, and that the "uncorrelated error" should really be thought of as the sum of a number of effects. Rounding error and "counting statistics" are two such effects. There are also effects which might be called "item effects", which are correlated for measurements of a particular item, but uncorrelated among items. An example is self-absorption in an NDA measurement. Self-absorption is a property of the individual item and influences the count rate; the variability of self-absorption values in a population of items is appropriately modelled in terms of an uncorrelated random effect, but cannot be estimated by the remeasurement of any one item.

\section{Rounding Error}

The error due to rounding is generally modelled as a random variable uniformly distributed over the interval in which rounding to the same value occurs. As the standard deviation of a uniformly distributed random variable is $R / \sqrt{12}$, where $R$ is the range, rounding to the nearest $G$ grams is modelled in terms of an absolute uncorrelated error with standard deviation .29G grams.

\section{NDA Counting Statistics}

The number of counts $C$ detected by an NDA device over a period of time $t$ is modelled as a Poisson-distributed random variable with mean $\mu=\lambda t$, where $\lambda$, the count rate, is related to (generally proportional to) the quantity to be measured and the efficiency of the detector. This randomness is a fundamental property of the physics of radiation. The standard deviation of the number of counts is $\sigma=(\lambda t)^{1 / 2}$, which can be estimated as $C^{1 / 2}$. If the amount of material is proportional to the count rate, then the uncertainty due to the effect of the Poisson counting statistics is proportional to the square root of the quantity of material, a situation which might be modelled either as an absolute or a relative uncorrelated effect. If it was determined to use the relative error model, the relative error standard deviation in the count is $C^{1 / 2} / C=C^{-1 / 2}$. If the SNM content of an item were given by $S=k C$, where $K$ is a constant, then a relative error model would look like 


$$
S_{\text {measured }}=S_{\text {true }}(1+\varepsilon)
$$

where $\sigma_{\varepsilon}^{2}=C^{-1 / 2}$.

Repeated Measurements of an Item

If one measures the value of an item $\mathrm{N}$ times the variance of the uncorrelated component of the measurement error can be estimated by

$$
\hat{\sigma}_{\varepsilon}^{2}=S^{2}=\left(\frac{1}{N-1}\right) \sum_{i=1}^{N}\left(x_{i}-\bar{x}\right)^{2}
$$

where $\mathrm{X}_{\mathrm{i}}$ is the result of the $\mathrm{ith}$ measurement. This statistic will not incorporate any information on "item effects" and so may seriously underestimate what one would like to use as an estimate of uncorrelated error. This can be remedied by performing repeated measurements for multiple items.

\section{Paired Data}

There are many situations in which the same characteristic is measured two times or more. If the two measurements are done by the same technique (e.g., replicate analyses of the same sample) an estimate of the uncorrelated variance is

$$
\hat{\sigma}_{\varepsilon}^{2}=\frac{1}{2 N} \sum_{i=1}^{N}\left(x_{1 i}-x_{2 i}\right)^{2}
$$

where $x_{1 i}$ and $x_{2 i}$ represent the $i$ th pair of measurements. This procedure is somewhat better than the previous one, because it at least measures more than one item, but it also fails to assess item effects.

One way to address the problem of item effects is to use two different measurement methods to measure a series of items and to use a Grubbs-type analysis (see [18] and [19]). The assumption is that the two methods will respond to different characteristics of the item, and thus the item effects will be independent for the two methods. Unfortunately, in some circumstances, these estimators have large uncertainties and may yield negative estimates of variances. Grubbs procedures are discussed extensively by Jaech [20]. In terms of using these procedures to estimate the characteristics of a single measurement system, they would appear to be safest when the standard deviation of the uncorrelated error of the measurement system of interest is large compared to that of the other system or to the variability of the true value of the items being measured.

The estimate of the uncorrelated error standard deviation of measurement system one is:

$$
\hat{\sigma}_{\varepsilon}^{2}=S_{1}^{2}-S_{12}
$$


where $S_{1} 2$ is the sample variance of the system "1" (one) measurements $\left(x_{11}\right)$, (equation (21)), and $S_{12}$ is the sample covariance:

$$
S_{12}=\frac{1}{N-1} \sum_{i=1}^{N}\left(x_{1} i-\overline{x_{1}}\right)\left(x_{2 i}-\overline{x_{2}}\right)
$$

\section{B. Specific Measurement Problems}

\section{Holdup}

Holdup problems are pervasive and sometimes play a dominant role in the behavior of inventory differences. Holdup is seldom easy to assess, and each holdup situation presents unique problems. From the point of view of variance propagation modelling, the interest is in the variability of the holdup from inventory to inventory, rather than in its absolute value. Relatively slow long-term buildup of the holdup will contribute to the long-term ID mean rather than to the ID variability. This variability should not include unusual incidents such as large spills or changes in state due to process upsets. Characterizing the holdup variability may be easier than solving other holdup-related measurement problems. There is an extensive literature on holdup problems, which was surveyed recently by Pillay in a special JNMM issue [21].

In MBAs where holdup is an important problem, locations where holdup can occur can be identified and ranked in terms of significance; as with other contributors to the ID uncertainty, the question of quantifying, estimating, or eliminating holdup in particular locations can be considered in terms of cost-effectiveness. For each location, a number of options are available (as suggested in [22]):

- Attempt to bring the process to a well defined state at inventory so that the holdup value for the inventory will vary as little as possible.

- Clear out the holdup to the extent possible at inventory, so that it is brought into a measurable form at that time.

- Measure the holdup in situ by means of NDA equipment.

- Model the behavior of holdup in terms of process parameters such as throughput and estimate the holdup based on the model.

- Use sampling techniques to select locations to measure holdup and extrapolate the results.

It is recognized, however. that many holdup problems remain difficult. If the absolute amount of holdup or its variability cannot be effectively eliminated the options are to measure by NDA, or estimate or model the holdup in some other manner. If there is a reasonable quantitative basis for the measurement or modelling, the holdup estimate can be booked and the uncertainty in the estimate can be used as a term in the variance-propagation model. 
Modelling will generally involve a comparison between values of some known parameter, such as throughput, and the "actual" value of holdup, as determined by recovery of the material or by some other method. The uncertainty of the holdup is estimated from the uncertainty in the model parameters and the residual variability in the model, as illustrated in the salt-bath example in section A of this chapter. This type of modelling is generally used in situations where there is a buildup of material over time until a cleanout of some kind occurs. The holdup on filters is another example of such a problem.

If (a), instead of accumulating, the holdup in a particular location can be assumed to vary in some manner around a steady-state mean (so that the holdup can be modelled as a sequence of uncorrelated random variables with the same standard deviation) and (b), good information is available regarding the inputs and outputs to the location so that an "inventory difference" can be formed around the location (which will not include the presumably unmeasurable holdup) then the variance of the holdup can be estimated from the covariance between the sequential IDs, $X_{i}$. This idea is considered in chapter $V$; the formula for the covariance between successive IDs is given in Table 5.1 as the value " $\mathrm{C}$ ".

Unmodelled holdup variability may be identified as a factor in the difference between a calculated and a historically observed inventory difference standard deviation. As mentioned previously, it is acceptable to make estimates of holdup variability in order to obtain consistency between historical and calculated values (although one should keep in mind that if most of the ID variability is to be explained as inventory-related, the historical ID serial correlation is expected to be strongly negative, as explained in chapter V). In such a case, the terms for the holdup variance should be identified with particular pieces of equipment or process locations where significant holdup is plausible; unless there is some reason to suspect otherwise the holdup terms corresponding to these distinct locations should be treated as uncorrelated.

\section{Sampling}

Generally speaking materiais that are not measured by NDA are measured using sampling in combination with a bulk measurement. This section will address the problems of the sampling of liquids, although sampling problems exist with respect to the sampling of powders and metals as well. At diffusion plants the sampling of UF6 gas from cylinders is a concern. Reference [23] gives a general overview of measurement control problerns in sampling. Generally the central question with respect to liquid sampling is how closely the elemental concentration of the sample matches the average concentration of the tank from which the sample was pulled.

The most common sampling situations are those involving uranyl- or plutonium-nitrate or chloride solutions. Liquid sampling systems in the nuclear industry must generate a sample at a point remote from the tank; to accomplish this sampling systems typically contain not only a sample line from the tank to the sample bottle, but return lines to effect recirculation and perhaps suction jet and air lift systems to move the liquid. Air lift systems assist in raising the liquid by introducing air into the sample line. 
The representativeness of the sample will depend on the degree of homogeneity in the tank and biasing effects introduced by the sampling system. Liquids sitting in tanks tend to stratify due to specific gravity variations; thus the homogeneity of a tank can never be assumed unless some type of mixing device is employed. The degree to which mixing occurs depends on the shape of the tank, the mixing mechanism, and the mixing time. Process tanks in the nuclear industry come in a variety of shapes, but long thin cylindrical tanks and flat rectangular ("slab") tanks are typical in situations requiring criticality safety; these thin geometries are also difficult to stir.

Mixing strategies include recirculation, sparging, and mechanical stirring. Procedures for sampling will generally specify that replicate samples be drawn and that strict guidelines be followed involving flushing of sample lines, recirculation, and so on. However, experience indicates that it is often difficult to obtain strict adherence to such guidelines, and in addition sampling systems are prone to various modes of failure which affect the samples. Any type of plugging affects recirculation or flushing, and leaks in air jet systems may expose the sample to excess dry air.

\section{Sources of uncertainty}

Sources of uncertainty in the liquid sampling process include:

- Lack of homogencity in the liquid in the tank.

- Biases introduced by the sampling system; for example concentration due to evaporation into the air supplied to the sample line in lift systems, or dilution from previous sample system flushes, or the mixing of "old" solution remaining in the sampling system with "new" liquid. Under most circumstances these effects are small, but malfunctions or improper operation of the sampling systems may exacerbate them. References [24], [25], and [26] provide examples and some data on these effects.

- Evaporation or other sample-handling effects after the sample is drawn and before it is analyzed.

The presence of solids in a tank will also lead to accounting uncertainties. However, the deposition of solids on the sides of a tank is really a holdup question, as sampling will never provide information on this material. The existence of solids suspended as particulates generally represents some sort of process problem and inust be eliminated by process techniques.

\section{Measurement Control}

A certain degree of control can be maintained over the sampling process by comparison of the lab concentration values of the replicate samples. However, lab values are often not available quickly enough to enable a resanıpling when a "bad" sampling occurs. For this reason, density measurements have become an important part of sampling system measurement control. The derisities of the replicate samples can be quickly compared on lab density meters; if the values are discrepant sampling is repeated. In addition, these lab densities may be compared with tank densities 
measured directly by electromanometer/bubbler probe systems. As the solutions involved become more dilute (so that the densities approach 1.0, i.e. highly aqueous), the sensitivity of these density tests to changes in concentration decreases; nevertheless, under certain conditions the density checks can be valuable down to concentrations of a few grams per liter.

Although general studies exist (see reference [27], for example) on tank mixing, the most effective way to establish the mixing properties of a particular tank is to perform a mixing experiment on the tank; this involves sampling the tank at fixed intervals for a period of time sufficient to bring the tank to a mixed state. Ideally a number of runs of this type should be performed. Each sample should be analyzed in duplicate for estimation and nuitigation of random analysis errors. It should be noted that tanks with poor mixing characteristics can take many hours to equilibrate. Such mixing studies can provide the basis for estimating sampling errors by analysis of the difference between the data at the time the tank will be sampled in practice and the data for complete mixing.

Bias may be introduced by evaporation and dilution of the sample as indicated in the second and third bullets above. These types of errors cannot be characterized by the kind of study described in the last paragraph because all data will be equally affected. One approach to the problem is to perform a special study of the properties of the tank and sampler using independent systems (see [12] p.115, for example). Once the tank has gone "hot" this becomes expensive, or may not be practical.

Density differences between lab and tank may also be used to estimate concentration sampling effects, provided the density data is available and sufficiently accurate. While there is no general relationship between plutonium (or uranium) concentration (because of the presence of other constituents of the nitrate solution) under specific process conditions density differences are good predictors of plutonium concentration differences due to dilution or concentration (see [2]). If density measurements are routinely made for control purposes, density-versus-concentration data are available with which to construct such a model. If, in addition, in-tank densities are available from electromanometer systems, inferences may be drawn regarding concentration or dilution between the tank and the lab sample.

\section{Statistical Modelling}

A detailed statistical model of liquid sampling uncertainties would address random effects due to tank sampling and analysis, correlated and uncorrelated effects due to incomplete mixing, and long-term effects such as consistent dilution or concentration by the sampling system. As mentioned previously, such detailed modelling may not be necessary in all circumstances.

Random errors in sampling plus analysis can be obtained by comparison of the analytical results from replicate samples via the pair-data statistic $\sigma_{\varepsilon}^{2}$ shown in equation (21) above.

In the present context

$$
\sigma_{r}=\sqrt{\frac{1}{2 N} \sum_{i=1}^{N}\left(x_{1 i}-x_{2 i}\right)^{2}}
$$


Here $x_{11}$ and $x_{21}$ are the replicate sample values and $N$ is the number of samples taken in a material balance period. Generally speaking there is no need to distinguish the two in the variance propagation model, provided it is kept in mind that both sources of uncertainty are captured in $\sigma_{\mathrm{r}}$.

Mixing experiments can generate data on (a), the variability of the concentration error for a given mixing time, and (b), whether there is any long-term effect. These experiments can be treateci using analysis-of -variance techniques (see [25], for example). A simple way to treat data from mixing experiments of the type described above is as follows. Suppose that $x_{1 i}$ and $x_{2} i$ are the replicate sample values for the ith run of a mixing experiment, drawn after 15 minutes of mixing (it has been determined that 15 minutes will be the mixing time required by the sampling procedure), and that y $1 \mathrm{i}$ and $y_{2 i}$ are the values at 2 hours (at which point it is assumed that the tank is thoroughly mixed). The random analytical error associated with an individual measurement can be estimated as:

$$
\sigma_{a r}=\frac{N^{-1 / 2}}{2} \sqrt{\sum_{i=1}^{N}\left\{\left(x_{1 i}-x_{2 i}\right)^{2}+\left(y_{1 i}-y_{2 i}\right)^{2}\right\}}
$$

where $\mathrm{N}$ is the number of runs. (This statistic in theory estimates the same kind of effects as the $\sigma_{\mathrm{r}}$ in the previous equation; however, in running this type of experiment, a different analytical technique may be used, and sampling will probably be done more carefully, so that this number will not reflect production sampling and sample-handling practices.)

The sequence of values

$$
z_{i}=\overline{x_{i}}-\overline{y_{i}}
$$

where

$$
\overline{x_{i}}=\left(x_{1 i}+x_{2 i}\right) / 2 \text { and } \overline{y_{i}}=\left(y_{1 i}+y_{2 i}\right) / 2
$$

represents the difference between the "partially" mixed state and the "completely" mixed state. The sample variance of the $z_{i}$

$$
\sigma_{z^{2}}=\frac{1}{N-1} \sum_{i=1}^{N}\left(z_{i}-\bar{z}\right)^{2}
$$

represents the variability due to imperfect mixing plus some random analytical error. The mixing variability alone is estimated by

$$
\sigma_{m r}=\sqrt{\sigma_{\mathrm{z}}^{2}-\sigma_{\mathrm{ar}}^{2}}
$$


(or zero if the difference is negative).

The average value of the $z_{i},(\bar{z})$, may show that there is a systematic effect in the mixing as well; in such a case, if a bias-correction is made (see section A), there will be an uncertainty assoctated with bias corrections of

$$
\sigma_{b c}=\sigma_{z} / \sqrt{ } N
$$

\section{Isotope Dilution Mass Spectrometry}

Isotope dilution mass spectrometry (IDMS) is used primarily for uranium and plutonium concentration determinations in nuclear material accountancy. It can also be used to determine isotope ratios for these types of samples.

Mass spectrometry is used to determine the relative numbers of ions of different masses in a sample. This information is referred to as the mass spectrum. The determination is accomplished by ionizing a portion of the sample and accelerating the resulting ions through a magnetic field. For the application under discussion, thermal ionization mass spectrometry is generally used; many other ionization techniques exist but will not be considered here. The elemental concentration (of plutonium in a nitrate solution, for example) can be found through analysis of the mass spectra of the sample before and after the addition of a "spike" of known isotopic composition and weight; thus the process consists of "wet chemistry" procedures (sample/spike preparation) and mass spectrometry procedures (obtaining the mass spectra). These two types of operations are generally performed in different places by different individuals.

In the calibration of the mass spectrometer there are two main concerns: fractionation and linearity. Fractionation relates to the composition of the ion beam produced by the ion source in the mass spectrometer. The thermal ionization process preferentially generates species of different masses, and this fractionation behavior depends on a number of experimental conditions. To compensate for this effect, a calibration procedure is used and experimental conditions are rigidly controlled. The calibration is performed by running standards of established isotopic composition.

Linearity describes the proportionality between mass spectrum peak heights and the corresponding isotope amounts originally present in the sample. Again, by running standards, the analyst can calibrate the instrument response to given amounts of isotopes.

There are three types of standards associated with these procedures: standards that are used to perform the instrument calibrations described above, standards that are related to the preparation of the spike, and standards that are used in measurement control programs for the method as a whole. Certified Reference Materials (CRMs) are available from New Brunswick Laboratory. Some facilitics use small, carefully measured quantities of these standard materials directly in their mass spectrometers for calibration. Other's make up secondary standards which they use in their day-to-day 
operations. These secondary standards must periodically be checked against tho original (primary) standard materials.

Spikes may be made directly from primary reference materials, or they may be prepared from other materials and standardized against the reference materials. Ideally, splkes consist of isotopes not present in the process sample; Pu-244 is commonly uivd as a splke.

\section{Sources of Uncertainty}

Uncertainties can arise in association with the use of standards, in chemical preparation steps and/or in the operation of instrumentation. Some uncertalnties will be captured by a measurement control program, some have to be estimated in other ways and some must be administratively controlled. Sources of uncertainty are:

- Uncertainty attributable to the preparation and maintenance of standards. Uncertainty can arise from any of the processes associated with the creation of the standard: weighing, dissolution, dilution, etc. There is of course some uncertainty associated with the primary reference materials themselves. Plutonium standards are especially difficult to maintain. In concentrated plutonium solutions, the plutonium causes dissociation of the water molecules, leading to gas generation which can pressurize (and possibly break) storage ampules; on the other hand, evaporation from unsealed containers will increase the concentration of the standard. If solids are used, the issue of complete dissolution for use arises.

- Interferences which occur because isotope masses are present in the spike that are also in the process sample, e.g., the same peaks will show up in a mass spectrum for Pu-238 and U-238 and for Pu-241 and Am-241.

- Changes in the fractionation bchavior of the mass spectrometer with time or environmental conditions.

- Differences in fractionation behavior between the standard used to calibrate the mass spectrometer and the unknown process sample because the chemical composition of the sample differs from that of the standard.

- Changes in the fractionation behavior of the instrument because changes occur in the way the sample is prepared for the mass spectrometer, or in the way the mass spectrometer is operated.

- Interfering elemental impurities (e.g, iron) are present in the process sample which, due to interactions with components of the mass spectrometer (the filament of the ion source) can lead to production of ions exhibiting masses equal to those of plutonium isotopes.

- Uncertainties or variabilities associated with sample/spike preparation. These include the usual uncertainties associated with wet chemistry: reading errors, 
wetghing errors, and so on. In IDMS of plutonitum solutions, it ts particularly important that the spike and sample are brought to the same oxldation state,

\section{Measurement Control}

Measurement control for IDMS should address three types of concerns related to the uncertainties described above:

(a) maintenance and calibration of the mass spectrometer itself,

(b) uncertainties associated with the IDMS procedures, and

(c) to the extent possible, uncertainties associated with the standards,

A measurement control program to control the IDMS procecture should be designed to catch errors in both the chemical preparation steps and the mass spectrometry step of IDMS; control samples should be run through the entire spiking/mass spectrometry procedure. These measurement control samples are synthesized to have a "known" concentration of uranlum or plutonium, but generally will not match the process material exactly in chemistry. Control samples are generally tested one per batch of "process" samples. Unless the measurement control program goes to considerable lengths to "matrlx match" the control samples with the process material there will be some differences in the response of the technique to the differing chemistry of the materials. The frequency with which control samples are analyzed in an IDMS system will be dependent on the type of instrument being used and on the schedule of process sample analyses being undertaken.

Usually different operators perform the chemical preparation and the mass spectrometry. It is difficult to make control samples blind for the chemist performing the preparation steps; for this reason, some operator errors may not be reflected in measurement control results. It is not difficult to make control samples blind for the technician running the mass spectrometer: the sample prepared by the chemist will be treated like any other sample when it comes to the mass spectrometry.

It has also been suggested [3] that comparative measurements be made on new concentration standard or spike working, solution batches against older solution batches before the older batches are discarded or used up entirely. This procedure provides some estimate of the uncertainties associated with standards preparation and storage described above.

The program described above will capture most of the uncertainties associated with opcrator error and instrumental variability. What will not be captured is some operator behavior due to nonblind standards, the effects of matrix mismatch, and some systematic effects in the proparation of standards.

Error Model

Data from the routine measurement control concertration measurements can be used to estimate uncorrelated and correlated effects using a bias correction as described in section A.4 of this chapter. Uncertainties in standards can be assessed from comparison of data from different batches of 
working standards, as descrlbed above, or from a considuration of the uncertainties lnvolved in the creation of the standard. The orror model in A.4 (equation [17]) can be elaborated to inclucte a term for a matrixumismatch offect:

$$
m y=\mu+\varepsilon_{1}+\eta^{\prime}+\eta^{\prime \prime}+\eta^{\prime \prime \prime}
$$

where the last term is a correlated offect estimating the matrix mismatch.

\section{Welghts}

Although weight moasurements are central to almost all nuclear material accountability systems, they generally do not play a dominant role in vartance propagation calculations. This ts simply because scales and balances in general perform so woll in comparison to other measurement techniques that weighing uncertaintles are not signifleant, even when applied to large throughputs or inventories. Weight measurement control and uncertalnty estimation are discussed in detail in [28], ANSI N15.18 - 1975, and [29]. For these reasons, the tople will be dlscussed only brlefly.

Weighng or mass determination systerns are traceable to the national system through mass standards which are generated by the National Burcau of Standards or which are traceable to such a mass standard. These objects are generally stainless steel, and are certified to a given uncertainty. These primary standards are used to assign values to test objects (also called secondary standards, checkweights, etc.) which are either used to calibrate mass measurement devices directly or to assign values to tertlary objects which are used directly. Gencrally speaking, one or two test objects are: weighed on each device elther once or twice per shlft. Corrective action (recalibration) is taken if the value generated does not fall within an acceptable range.

Uncertainties in weight determination may arise from the inherent variability of the Instrument, from calibration uncertainties (due to uncertainties in standards), from rounding error, from operator practice (for example some scales respond to the location on the platform at which the object is placed), environmental effects. Buoyancy (of air) becomes a significant factor if the density of the unknown object is very much different from the density of the test object. For this reason and others special Replica Mass Standards in the form of UF-6 cylinders have been created for control of UF-6 weighings.

Reference $|30|$ describes two methods for estimating random uncertatnties for mass measurements, one using multiple woighings, and one using paired weighings of production objects. The estimate of random uncertainty should not be less than the uncertainty due lo scale rounding (discussed in section A.5). Repeated weighings of a single object may often round to identical values and so not produce a useful result.

In terms of blas effects, any uncortainty in the value of a test object or mass standard will, of course, propagate as a bias effect through all of the mass determinations affected by calibrations using; that standard. Thus a calibration using a tertiary standard will load to a bias term in the error 
propagallon roflecting the uncertalnty in the primaxy standard and the uncertainty in the deterninations of the values of the secondary and tertary values as well:

$$
\sigma_{\mathrm{blas}}^{2}=\sigma_{1}^{2}+\sigma_{2}^{2}+\sigma_{3}^{2}
$$

Estimates of the standard devlations of correlated and uncorrolated offects can often bo checked by comparison of the weights of production objects measured first on one scale and then (after a transfer) on another scale. Such data are often generated as a part of normal operations; as such they will probably reflect the actual system performance better than results of check weighings. Reference $|12|$ provides addittonal methods for estimating the uncertainty In mass standards.

Many mass measurements enter the accountancy system as part of a gross minuis tare subtraction. Unless there is information to the contrary, it is not approprtate to suppose that elther the random or blas effects will cancel in this subtraction, so that the uncertainty ior the difference is:

$$
\begin{aligned}
& \sigma_{r \text {, difference }}^{2}=\sigma_{\text {r,gross }}^{2}+\sigma_{r \text {, tare }}^{2} \\
& \sigma_{b, \text { difference }}^{2}=\sigma^{2}{ }_{b, \text { gross }}+\sigma_{b \text {,tare }}^{2}
\end{aligned}
$$

This point is mentioned because some variance propagation algorithms may cause correlated effects to cancel rather than add if not handled correctly.

\section{Non-Destructlve Assay}

NDA techniques are used to determine the fisstle content of discrete items, or samples of bulk material, by the observation and measurement of spontaneous or stimulated nuclear radiations, such as neutrons or gamma rays, or by the measurement of the (Indirect) heating due to alpha (or other) particles.

Gamma ray measurements may involve identification and intensity measurement of gamma and $x$-ray lines, measurement of the ratlos or correlations of particular lines, and attenuation and absorption determinations related to the intensity measurements. Since gamma rays are sitrongly absorbed by dense materials these methods are applicable to limited types of sample configurations, compositions and sizes. Gamma spectroscopy is useful for the determination of the isotopic fractions in uranium and plutonium, which must be known for the use of neutron and calorimetric techniques.

Neutron methods involve the counting of coincident and non-coincident neutrons in order to determine the amount of SNM in the item examined. Non-coincident counting is useful for process monitoring, and for certain rough indications of the presence of fissille matertals. However 
for accurate measurements, both kinds of counting must be used, with the colncidence counting playing the prectominant role.

Calorimetric methods depend on the internal heating of the plutonlum-bearing sample by its radioactive decay products. Due to thermal inertia the present production methods are lengthy, usually several hours. The thermal effects are Independent of the fine detalls of the nuclear interactions within the sample, but they may be affected by chemtcal reactions.

\section{Sources of Uncertainty}

The sources of error thus fall into the following classes:

- those assoclated with the direct instrument characteristics, such as lincarity, nolse, stabillty, etc.;

- those connected with amblent effects, such as background and environmental variability;

- those due to uncertainties in calibration model and standards;

- those due to the plyysical and calculational structure of the measurement model;

- those due to the characteristics of the items measured that are not reflected in the standards and thus not taken into account in the callbration procedure. In the case of gamma can-scan type equipment, this characteristic may be self-absorption; in the case of neutron coinctdence counting, it may be multiplication or the presence of molsture, fluorine or other impurittes;

- In the case of neutron colncidence counting and calorimetry, the uncertainties connected with the associated gamma determinal on of the isotopics.

\section{Measurement Control and Modelling}

Measurement control for most NDA systems involves calibration, periodic recalibration, and measurement of standards. In some cases where materials are uniform and well-characterized, such measurement control programs will be an adequate basis on which to estimate measurement uncertainty, and the type of models described in sections A.1, A.2, and A.4 of this chapter apply. In some cases, particularly the application of gamma scanning and neutron coincidence counting to scrap and waste materials, neither the calibration procedure nor the standards will reflect the variability in characteristics affecting the measurement that exists in process materials. If the process material can be characterized with respect to the interfering characteristics then it may be possible to fabricate appropriate sets of standards.

If calibration and measurement control data do not reflect true measurement uncertainty, it may be possible to use another well-controlled measurement method to measure production items on a sampling basis; these values may be used in effect as measurement-control data. Sometimes scrap items may be recovered in such a way that the recovery values are accurate enough to use as a comparison against original NDA-measured values. 
Low-level waste matertals (usually fncluding wipes, bootles, etc.) In large drums are often a measurement control problem; the only measurement techniques which are applicable are gammascanning and (for plutonlum) noutron colnddence counting. The ltems tend to be heterogeneous with respect to important effects (such as density or self-absorption for a gamma mensurement). Possible stratogles involve categorizing items by content or by assay (and treating each category with distinct calibratton curves). In some locations gamma and neutron assays are averaged. In most cases it is only necessary to get a very crude estimate of these uncertatnties because of the low item (SNM) content in drums of waste material. 


\section{Historical ID Data and Control Limits}

At the end of chapter II the following general form was suggested for the calculation of control limits:

$$
A+B \pm C D \sigma_{I D}
$$

where the constants $A, B, C$, and D have been described in Chapter IID above.

This chapter addresses the following questions:

(a) the use of a mean value term (the "A"in formula (1));

(b) the use of a correlation coefficient (relating to the "B" and " $\mathrm{C}$ " terms); and

(c) the determination of the consistency of the variance propagation model with historical ID data.

The following courses of action are recommended with regard to these issues.

- The observed historical data are consistent with the variance propagation model when the historical and calculated values for the ID standard deviation and serial correlation agree within statistical uncertainty, when the historical data and model have been appropriately adjusted. In many cases this uncertainty may be quite large.

- A mean-value term should be included if the historical IDs show a statistically significant non-zero mean over an extended period of time. This approach has been suggested in a recent OSS memo [5].

- From a purely technical point of view, it is valid to either use or omit the terms dealing with correlation; the choice is therefore a matter of judgement. It is suggested that these terms be used when the correlation $\rho$ is large and well-known, and omitted (i.e., set $\rho=0$ ) otherwise.

The remainder of this section discusses these points. It should be kept in mind that in practical situations small sample sizes, changing facility conditions, and deviations from the assumptions generally made as a basis for statistical theory will mean that a good deal of judgement must be exercised in the application of the statistical tests and estimates.

A. A Model of the ID Sequence.

A simple statistical model of the inventory difference is useful as a basis for discussing the properties of a sequence of historical IDs. The model is defined mathematically as follows:

$$
X_{i}=\delta_{i}-\varepsilon_{i-1}+\varepsilon_{i}+\mu
$$

where 


$$
\begin{array}{ll}
X_{i}= & \text { the ith ID } \\
\delta_{i}= & \text { the error in the net flow (additions - removals) for period } \mathrm{i} \\
\varepsilon_{\mathrm{i}}= & \text { the error in the beginning inventory for period } \mathrm{i} . \\
\mu \quad= & \text { an average, long-term value of the ID due to unknown biases, } \\
& \text { unknown flows, etc. }
\end{array}
$$

It is assumed that all the errors are uncorrelated, and all have mean zero. These are reasonable assumptions, as most ID components are measured by different methods, and many of the existing sources of correlation do not contribute to observable ID variability. For example, the fixed component of a holdup quantity disappears in the BI-EI (beginning inventory minus ending inventory) subtraction, and monotonically increasing holdup is incorporated into $\mathrm{m}$; on the other hand, the type of behavior that cannot be reflected in the above model is buildup which gradually increases until it is cleaned out once every other material balance period.

The variance of the ID in the above model is

$$
\operatorname{Var}\left(X_{\mathrm{i}}\right)=\sigma_{\mathrm{ID}}{ }^{2}=2 \sigma_{\varepsilon}^{2}+\sigma_{\delta}^{2}
$$

The covariance between successive IDs is $\sigma_{\varepsilon}^{2}$, while the covariance between any non-adjacent IDs is zero. 'The correlation between successive ID's is

$$
\rho=-\frac{\sigma_{\varepsilon}^{2}}{2 \sigma_{\varepsilon}^{2}+\sigma_{\delta}^{2}}
$$

Clearly if the flow variance $\left(\sigma_{\delta}^{2}\right)$ is very much greater than the inventory variance $\left(\sigma_{\varepsilon}^{2}\right)$ then $\rho$ is close to zero; in this case the flow errors are dominant, and there is very little correlation between successive IDs. The opposite case, when $\sigma_{\varepsilon}{ }^{2}$ is much greater than $\sigma_{\delta}{ }^{2}$, is inventory dominated and correlation between successive IDs is close to $-1 / 2$.

The variance of a sum of IDs

$$
\operatorname{Var}\left(\sum_{i=1}^{n} X_{i}\right)=2 \sigma_{\varepsilon}^{2}+n \sigma_{\delta}^{2}
$$

In the flow-dominated case, the variance grows linearly with $\mathrm{n}$; in the inventory-dominated case, there is (initially) little change in variance with $n$. The variance of the ID mean is 


$$
\operatorname{Var}(m)=\operatorname{Var}\left(\sum_{i=1}^{n} \frac{x_{i}}{n}\right)=\frac{2 \sigma_{\varepsilon}^{2}+n \sigma_{\delta}^{2}}{n^{2}}
$$

The quantities $m, \sigma_{\mid D}{ }^{2}$, and $\rho$ can be estimated from an observed historical ID sequence $\left(X_{1}\right.$, $\left.\mathrm{X}_{2}, . . \mathrm{X}_{\mathrm{n}}\right)$ using the statistics which are summarized in Table 5.1 .

\section{B. Comparison of Historical ID Data and Variance Propagation Results}

Variance propagation results can be checked against historical ID data by comparison of the historically observed values of $\sigma_{\mathrm{DD}^{2}}{ }^{2}$ and $\rho$ (see Table 5.1) with the corresponding values from variance propagation computations. This is essentially a test-of-hypothesis situation. To make this comparison as legitimate as possible, the following factors should be considered:

- The historical ID data should reflect the same set of adjustments as are assumed in the variance propagation model.

- The historical ID data should be corrected, to the extent possible, with respect to anomalous occurrences that are outside the scope of the model. The simplest example of such an occurrence would be an item missing at the end of one inventory period but recovered the next. A similar situation might involve a process upset in which material spilled, condensed, or solidified out was recovered after having been effectively missing at one inventory.

- The values in the variance propagation model (for flow and inventory quantities, for example) should reflect the appropriate historical conditions. It may well be that the model may have to be altered to reflect historical conditions appropriately. Obviously, there may be a point in time when historical conditions are sufficiently different from present ones that they cease to be relevant.

In spite of these precautions, the comparison between historical and variance-propagation results may not be very exact, depending on how many IDs are used in the the calculations.

In particular, any estimates of the serial correlation coefficient $r$ will produce very wide confidence bounds. Confidence intervals for correlation coefficients are shown in Table 5.2 (from reference [31]; strictly speaking these bounds are derived on the basis of points independently chosen from a two-dimensional normal distribution rather than from pairs of sequential points in a time series). Because it is likely that only broad comparisons can be made using the correlation coefficient, it is suggested that only a qualitative judgement be made. One ought to be able to distinguish heavily inventory-dominated situations from heavily flow-dominated ones; when the historical data indicate one situation, and the error-propagation results indicate the other, some explanation should be sought. 

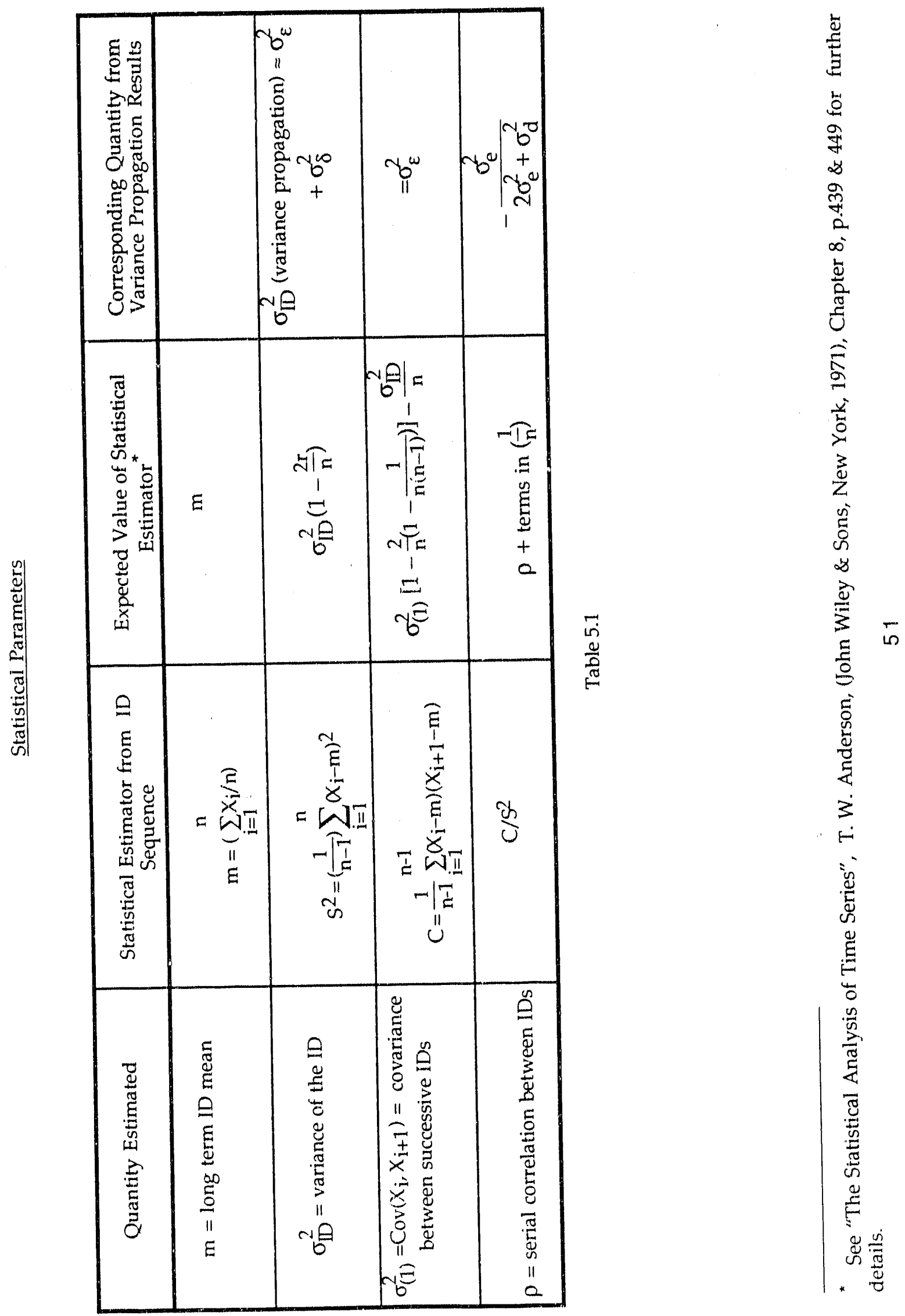
Comparison of the historical and error-propagated ID standard deviations may yield more quantitative answers. The simplest way to do this, assuming that a recent time period exists for which the error-propagated standard deviation is approximately constant, is to look at the ratio

$$
\frac{\sum_{i=1}^{n}\left(x_{i}-m\right)^{2}}{\sigma_{I D}^{2}}
$$

and to treat it as a chi-square distribution with n-1 degrees of freedom. Statistics texts commonly tabulate confidence intervals for chi-square variances. The cited Table 5.2 gives examples of $95 \%$ confidence limits for various values of $n$ :

\section{Conficience Limits for Different Values of $n$}

\begin{tabular}{|ccc|}
\hline $\mathrm{n}$ & lower limit & upper limit \\
\hline 5 & .83 & 12.8 \\
10 & 3.25 & 20.5 \\
15 & 6.26 & 27.5 \\
20 & 9.59 & 34.2 \\
25 & 13.1 & 40.6 \\
\hline
\end{tabular}

Table 5.2

Obviously, if the ratio is higher than the upper limit, then the historical value significantly exceeds the variance-propagated, and if the ratio is lower than the bottom limit, the opposite is true. Note that if the above limits are translated into the terms of a $95 \%$ confidence limit on a standard deviation, even for $\mathrm{n}=25$ the limits are approximately $\pm 26 \%$. The above test is applicable if the entire sequence of IDs are supposed to have approximately the same value for $\sigma_{\text {ID }}$ (and is strictly valid only for situations in which $r$ is close to zero). If historical conditions vary from period to period, so that a sequence of variance-propagated $\sigma_{I D}$ 's are identified as $s_{i}$, the expression

$$
\sum_{i=1}^{n} \frac{\left(x_{i}-m\right)^{2}}{\sigma_{i}^{2}}
$$


can be used instead of the previous one.

As noted above, when there is correlation between IDs $(r \neq 0)$, these statistical tests are no longer strictly valid, as the distribution of these statistics becomes complex. The effect of the negative correlation according to both theory and calculation is to increase slightly the variance of statistics such as

$$
\sum_{i}\left(X_{i}-m\right)^{2}
$$

Monte Carlo calculations indicate that this increase is small (less than 10\%) for $r$ in the range $[0,-0.25]$ and between 10 and $20 \%$ in the range $[-.025,-0.5]$. An approximate approach to the problem of correlation is to ignore it unless it is in the latter range, in which case the confidence interval can be lengthened by about $20 \%$.

As a crude rule of thumb, the relative standard deviation of the estimate of the standarid deviation given by

$$
\hat{\sigma}_{I D}=\sqrt{S^{2}}=\sqrt{\left(\frac{1}{n-1}\right) \sum_{i=1}^{n}\left(X_{i}-m\right)^{2}}
$$

is approximately $\sqrt{ }(2 n)$. In other words, if the ID standard standard deviation is estimated by the above formula to be $1.3 \mathrm{~kg}$. using 25 IDs as data points, the relative error is $1 / \sqrt{ }(50)$ or 0.14 . If we use two standard deviations as a crude $95 \%$ confidence interval, the confidence interval on $\mathrm{S}_{\mathrm{ID}}$ can be quoted as approximately $1.3 \pm 0.36 \mathrm{~kg} .: 0.36=(2)(1.3)(0.14)$. (This value is approximately $\pm 28 \%=$ $0.36 / 1.3$ which is reasonably consistent with the value quoted above based on the $95 \%$ chi-square limits.) In the situation where $r$ is large these limits could be expanded by $2.0 \%$ to $\pm(0.36)(1.2)= \pm 0.43$.

\section{Assessing Long-Term ID Averages}

There is a considerable literature on the detection of the removal of material from accounting systems over multiple time periods or from multiple MBAs (see [32], [33], [34], and [35]). A review of this topic is beyond the scope of this document, except to note that the best statistic for detecting a constant removal of material over a fixed time period is a simple average, or equivalently a cumulative sum. When dealing with material balance periods of a month or more, it is unlikely that one will have a large number (20 or 30 ) data-points in which the system can be said to be (even approximately) in a steady-state condition. The most straightforward statistic that can used to estimate, and assess the significance of, the long-term mean is the simple average " $\mathrm{m}$ " of Table 5.1, taken over recent ID's for a period of time in which conditions are reasonably close to present 
conditions. The determination of what this time period should be must be based on a practical knowledge of facility conditions, In some cases there may be very little relevant historical data; in this case the mean is taken to be zero.

The easiest and most conservative way to assess the significance of the value of " $m$ " is to use the "T statistic":

$$
\mathrm{T}=\frac{\mathrm{m}}{\sqrt{\mathrm{S}^{2} / \mathrm{n}}}
$$

where $S^{2}$ is given in Table 5.1 (notice $S^{2}$ is the unbiased version of the variance estimator); the $T$ statistic has $n-1$ degrees of freedom. Confidence limits are available as a function of the number of degrees of freedom in most statistics texts; if zero is not included in the $95 \%$ confidence interval, one concludes that a long-term trend exists, and uses the value of $m$ as a long-term ID mean.

These confidence limits are based on the assumption of independence between the observations $X_{i}$; any negative correlation among the $X_{i}$ will tend to reduce the variance of the average and thus increase the true statistical significance of the result. Therefore a T-value which is computed as being significant will be truly significant regardless of negative correlation.

On the other hand, a T-value computed to be insignificant when correlation is present may in fact be truly significant. One way to assess this is to look at the ratio.

$$
\frac{m}{\sqrt{\frac{2 \sigma_{\varepsilon}^{2}+n \sigma_{\delta}^{2}}{n^{2}}}}
$$

where the denominator is the standard deviation of the ID mean as computed from variancepropagation results, as described in section A. In theory, this statistic is normal with standard deviation 1, so that an absolute value exceeding approximately 2 indicates significance. To use this formulation, the variance-propagation and historical results should be in good agreement.

\section{The Use of Correlation Coefficients}

In situations when the inventory-difference is heavily inventory-dominated, a pattern will emerge where a large positive ID will be followed by a negative one, and vice versa. Use of the " $B$ " and " $\mathrm{C}$ " terms in formula (1) in section 3.A causes the control limits to shift back and forth in the same manner. They are control limits based on the conditional mean and standard deviation of the present ID given the previous ID. In theory, the same probability of a false alarm (type I error) exists whether one uses information regarding the current ID or not; or in other words, whether or not one ignores the correlation. In the case where $p$ is small there is of course no practical difference in any case. Where $\rho$ is large (near -0.5 ), ignoring the correlation will result in a situation where alarms 
are clumped; one alarm will tend to be followed by another, both alarms probably caused by one bad inventory value. For this reason, when both the variance propagation and the historical $1 \mathrm{D}$ sequence indicate a large negative correlation, use of the $B$ and $C$ terms is advisable. The quantity $p$ can be estimated from historical data or from variance-propagation results as indicated in Table 5.1. 


\section{Variance Propagation Algorithms}

This chapter discusses calculational methods for varlance propagation. A number of methods for doing these calculations are explained in texts (such as [1] and [12|) or realized ats computer software. All these methods use different approaches and result in slightly different algorithms and formulas. Despite the availability of these methods, there are advantages to writing software specific to a given application. The first section of this chapter describes a very general method by which variance-propagation furmulas or algorithms may be created from first principles based on the concepts described in Chapter III and IV. The second section describes an easilyconstructed variance propagation model sultable for simple accounting situations, and then describes two existing computer codes that considerably extend this type of model.

\section{A. A General Variance-Propagation Formula}

Variance propagation models and algorithms take many different forms, but most of the formulas can be derived from a general expression based on the idea of random effects. This expression is described here and derived in Appendix A. Many of the complexities which arise in formulating the more elaborate models derive from the chore of defining which error effects are associated with which items.

The inventory difference may be written as

$$
I D=\sum_{i} s_{i} f_{i}\left(m_{i 1}, m_{i 2}, \ldots\right)=\sum_{i} s_{i} f_{i}\left(m_{i}\right)(1)
$$

where $i$ is an item index, $s_{i}$ is the sign with which the value of the item enters the material balance, and $f_{1}$ is the item function, based on observed values $m_{i 1}, m_{12}$, etc. (sce III.A for definitions of these terms). A static inventory item may enter this sum twice. Each measured value is related to an absolute or relative error model, and each error in the error model is associated with a random effect $E$ as indicated in Figure 6.1. As shown in this figure, errors may be replicated a number of times when they represent correlated effects. (It is convenient, and very plausible, to assume that a given error shows up only once for each item).

If $\sigma_{\mathrm{E}}{ }^{2}$ is the variance associated with effect $\mathrm{E}$, the variance of the ID may be 


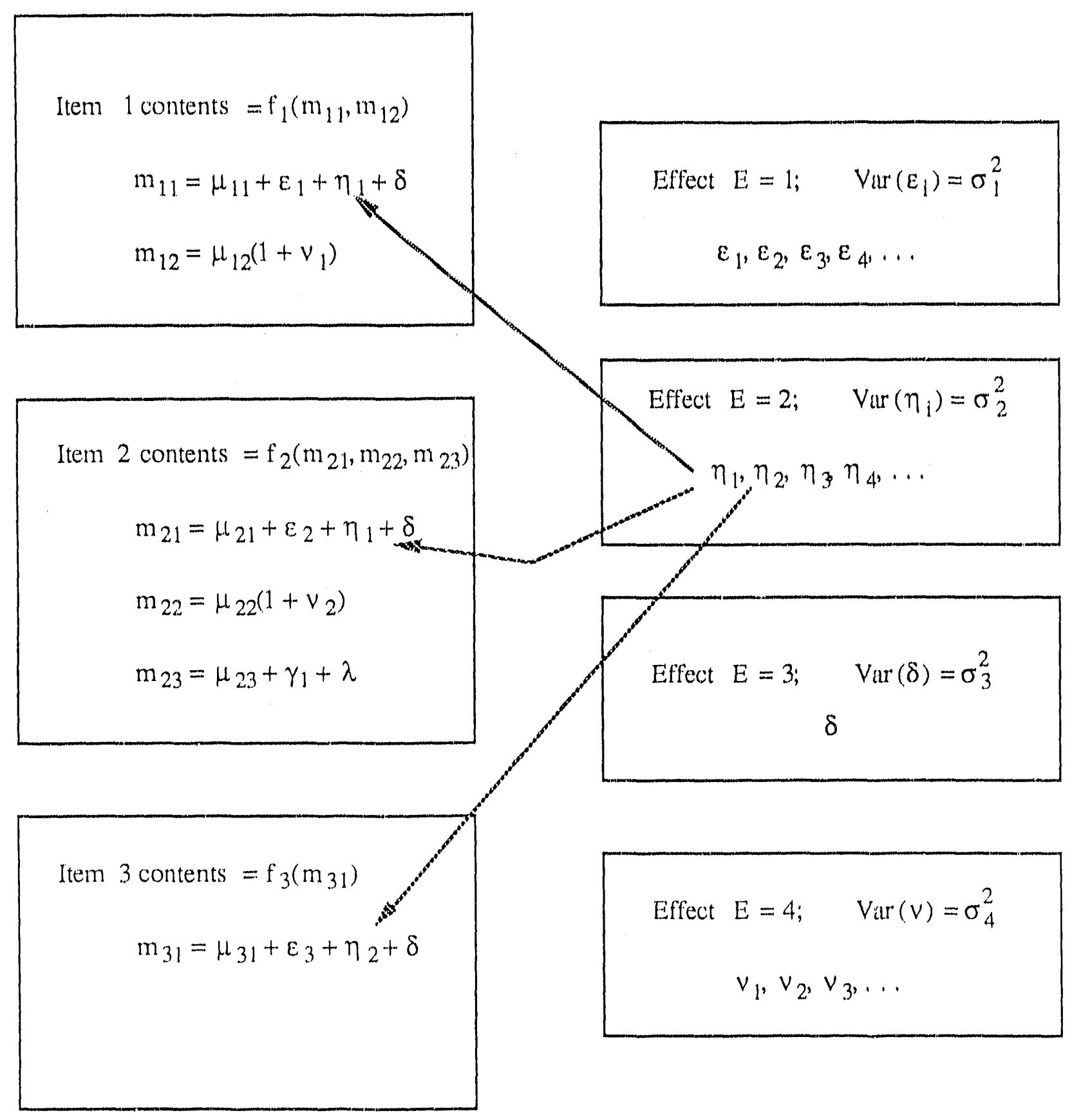

Figure 6.1 
calculated as follows:

$$
\begin{gathered}
\sigma_{I D}{ }^{2}=\sum_{\mathrm{E}} \mathrm{V}_{\mathrm{E}} \\
\mathrm{V}_{\mathrm{E}}=\sigma_{\mathrm{E}}^{2}\left[\sum_{\xi \in \mathrm{E}}\left(\sum_{(1, j)) \rightarrow \xi_{0}} \mathrm{II}_{\mathrm{H}}\right)^{2}\right]
\end{gathered}
$$

where $\xi$ reprosents the error belonging to the effect $E,(i, c, \xi \rightarrow \varepsilon, \delta, \eta, v, \ldots$ as approprtate)

$$
\mathrm{H}_{i j}=s_{i} \mathfrak{F}_{i}^{(j)}\left(\mathrm{m}_{1}\right) \Gamma_{i j}
$$

and $\mathrm{V}_{\mathrm{E}} \quad$ = the total variance contribution associated with effect $\mathrm{E}$,

$$
f_{i}^{(j)}\left(m_{1}\right) \quad=\text { the partial derlvative of } f_{i} \text { with respeet the fth argument, evaluated }
$$
at $\underline{m} 11$,

and I"if $\quad=1$ if the measurement model for the flh measurement is absolute, or $=\mathrm{m}_{\mathrm{ij}}$ if the model is relative.

The summation notation can be described as follows: for each error $\xi$ belonging to an effect E, find all Items i such that the error $\xi$ appears in an error model for that item (for some measurement $j$ ). For each such item $i$, compute $\mathrm{H}_{\mathrm{ij}}$. Sum the $\mathrm{H}_{\text {if }}$ values over all items for a partleular $\xi$, and square the result. Sum the results for all $\xi$ relating to a particular $\mathrm{E}$ to get the total for $\mathrm{E}$ (in other words, $V_{\mathrm{B}}$ ). Sum all $V_{E}$ values to get the total standard deviation.

In the case of an effect which is uncorrelated between items, one error corresponds to oric ltem, so) the expression reduces to

$$
V_{E}=\sigma_{E}^{2}\left(\sum_{i} H_{i j}{ }^{2}\right)
$$

For a totally correlated effect there is only one error but many llems, so the result is

$$
V_{E}=\sigma_{E:}^{2}\left(\sum_{i}^{-1} H_{i j}\right)^{2}
$$

Example 6(a):

Consider the variance due to the effects ew and hw in example 3.6 (chapter 1I1). In this example we have 


$$
f_{1}\left(w_{1} e_{1} p_{1}\right) \quad=w_{1} e_{1} p_{1}
$$

and

$$
f_{1}^{(1)}\left(w e_{i} p_{i}\right)=e_{i} p_{i}
$$

Slnce the error model is relative, wo have

$$
\Gamma_{11}=W_{1}
$$

so according to equations (5) and (6), the vartances assoctated with $\varepsilon_{W}$ and $\eta_{W}$ are

$$
\sigma_{\varepsilon, w}^{2} \sum_{l=1}^{N}\left(w \mid e_{1} p_{1}\right)^{2} \Rightarrow N x^{2} \sigma_{\varepsilon, w}^{2}
$$

and

$$
\sigma_{\eta, w}^{2}\left(\sum_{i=1}^{N}\left(w_{j} e_{i} p_{i}\right)^{2} \approx N^{2} x^{2} \sigma_{\eta, w}^{2}\right.
$$

as indicated in example 3.6.

Example 6(b)

Suppose an item value is calculated as some power of a measured value (as in reference $[36\}, p, 8): f_{1}\left(m_{1}\right)=m_{1} P$ (there is only one measured value $m_{1}$ for each $\left.1 t e m i\right)$ and $m_{1}$ has uncorrelated and correlated effects $(E$ and $\eta$ ) associated with it in a relative error model.

$$
\begin{aligned}
& f_{1}^{(1)}\left(m_{1}\right)=p_{11}^{(p-1)}, \\
& \Gamma_{1}=m_{1},
\end{aligned}
$$

and

$$
\mathrm{H}_{\mathrm{i}}=\mathrm{pm}_{\mathrm{i}}^{\mathrm{p}}
$$

The varlance due to the uncorrelated effect $\varepsilon$ is

$$
\sigma_{\varepsilon}^{2} \sum_{i}\left(p m_{i}^{p}\right)^{2}
$$


while the varianed due to $\eta$ is

$$
\sigma_{\pi}^{2}\left(\sum_{1} p_{1} m^{p}\right)^{2}
$$

'These are essentially the formulas that come into play in [36].

If ono can describe the accounting system in the form of equation (1), and the error in the accounting system using orror models and random effects as described, then formulas (2)-(4) may help provide gudance as to how to set up a vartance propagation calculation.

\section{B. Variance Propagation Algorithms}

Most calculational methods (whether described in a text or implemented as software) are directed at particular applicalions and are compromises between easc-of-use and modelling flexibility. Circumstances which will influence the way in which a calculation model is chosen or developed are the following:

- The level of detail of the data avallable to the calculation.

Detalled error models involving distinctions among instruments or callbration pertods will require specific information on which instruments made which measurements, or at least general inforrnation on the distribution of numbers of measurements made by instruments.

- The type of instruments modelled and the level of detall at which statistical modelling takes place.

In most circumstances it is adequate to model an item quantity as a product of two or three values (concentration $\times$ volume, parity $\times$ weight), but occasionally more detalled modely may be used, employing complex formulas involving inverses or exponents.

- The type of correlations which must be modelled.

Many models allow only totally uncorrelated or totally correlated effects, ("random and blas"), and may not be flexible in terms of the range of correlation that can be attributed to the totally correlated effects. For example, the effect due to the uncertainty in a standard may be appllcable to a number of instruments (all calibrated with the standard) but the uncertainty due to a calibration procedure is applicable to cach instrument separately. Stich distinctions may or may not bo feasible with a given code.

- Absolute or relative error models may be appropriate in different circumstances.

In many cases the variabllity in the measured value is small enough so that it makes very little difference, and in such cases relative error models are generally 
used, as uncertainties are often quoted a.s percents, and as the variance-propagation formulas are easier to program.

- It may be desirable to employ error models with a number (more than two) of elfects.

- It may be approprtate to handle items in the material balance or an individual basis (using the actual observed values of volume and concentration of a process tank, for example), or on an aggregate basts (using the total number of cans of powder and the average weight and parity values of the cans, for example).

T'wo Simple Models

Described below are two simple models that are very easily programmed, and two more complex models which are already available as code.

A very simple model is based on the assumption that the matertal balance components can each be divided into a number of strata, and that each stratum is measured independently of the others. A product model for the tem function for the content of each item is used, and the error model for each measurement is a relative model involving a completely uncorrelated effect and a completely correlated effect. This situation was described in example 6 of Chapter III. This type of model is used for theoretical calculations, but in practice the restriction that measurements are uncorrelated between strata may be untenable.

Figure 6.2 illustrates a model in which measurement methods are described independently of the strita. As before, the error model for each method is a relative model with totally correlated and totally uncorrelated effects. The Item function is assumed to be in the form of a product (two factors are listed, but there could be any number of such factors), Effects associated with different measurement methods are assumed to be uncorrelated. Associated with each measurement method is a list of the strata to which these methods apply.

The variance propagation calculation is quite simple, as shown in Figure 62 . In fact, because one can define strata to be as small as a single item, and one can assign methods in an arbitrary way to strata, this formulation can generate a fairly elaborate model. It will not handle additive error models or complex ttem functions, however.

\section{AMASS}

The AMASS [37] calculation is very much like that described in the above paragraph, but also implements a calculation for partially correlated effects. AMASS again in effect uses a product item function and a relative error model. In AMASS, the error model can include a number of totally correlated and partially correlated effects, along with an uncorrelated effect. Control over the range of correlation for totally and partially correlated effects is established using a hicrarchy of categorics which include the type of meast.rement (e.g. bulk or analytical), the "source", which could, for example, identify a particular instrument, and the "condition" of the source, which might change with shift or recalibration. Changes in "condition" are used to implement partially correlated effects. 
It is possible, but not easy, to implement absolute error models using AMASS. No provision is made for item functions which are not simple products.

\section{Summary of a Simple Method-Oriented Variance Propagation Model}

\section{Stratum Data}

$\mathrm{N}_{\mathrm{s}}: \quad$ number of items in the stratum

$S_{S}: \quad \pm 1$ depending on whether the stratum contributes positively or negatively to the ID

$P_{5}: \quad$ grams SNMI/gram weight: average per item

$W_{5}$ : average weight per item

$G_{\mathrm{S}}: \quad$ average item content $=\mathrm{P}_{\mathrm{S}} \mathrm{W}_{\mathrm{S}}$

\section{Measurement Method Data for Method M}

$\sigma_{r, M}:$ relative uncorrelated error standard deviation

$\sigma_{\mathrm{S}, \mathrm{M}}$ : relative correlated error standard deviation

$I_{M}$ : list of all strata for which $M$ is the measurement method.

Variance associated with method $M$ is salculated as:

$$
V_{M}=\sigma_{s, M}^{2}\left(\sum_{s \in I_{M}} S_{s} C_{s} N_{s}\right)^{2}+\sigma_{r, M}^{2}\left(\sum_{s \in I_{M}} N_{s} C_{s}^{2}\right)
$$

where $s \in I_{M}$ indicates a sum over all strata $s$ in the list $I_{M}$.

The total ID variance is the sum of the above expression over all methods.

$$
\sigma_{\mathbb{D}}^{2}=\sum_{M} V_{M}
$$

Figure 6.2 


\section{MAWST}

MAWST is a variance-propagation code which can be used for statistical modelling at a very detailed level. It supports absolute and relative error models, and it supports item functions which are fairly general in nature. The form of the allowed item function is

$$
\sum_{i} s_{i} \prod_{j}\left(m_{i j}\right)^{p i j}
$$

where $s_{i}$ is $\pm 1, m_{i j}$ are measured values or constants, and $p_{i j}$ are exponents. A second level of modelling allows the computation of complicated functions of item functions.

The error model for the $\mathrm{m}_{\mathrm{ij}}$ incorporates two effects, labeled $\mathrm{e}$ and $\mathrm{h}$. The correlation characteristics of these effects are controlled by assigning to each measurement three indices: MID, REC, and TSTP (for measurement instrument ID, recalibration period and time-stamp). The effect $e$ is correlated over all measurements in which all three indices match; it is basically an uncorrelated error. The effect $h$ is correlated over all measurements for which MID and REC are identical; thus $h$ can play the role of a partially or totally correlated effect, depending on how REC is treated. Static inventory need not be stripped out of the data before submission to MAWST; presumably if an item is on static inventory, all three indices will be identical and the code will appropriately cancel out any contribution to the ID from the item.

Additional effects (in addition to $e$ and $h$ ) for a particular measurement are not formally supported by the calculation, although they can be inserted by incorporating into the model additional (fictitious) measurements. 


\section{References}

[1] Statistical Methods for Nuclear Material Management, W. M. Bowen and C. A. Bennett, Editors, NUREG/CR-4604, PNL-5849, (U. S. Nuclear Regulatory Commission, Washington, DC, December 1988).

[2] C. Bingham, "D.O.E. Target Value Program", prepared for DOE/OSS by New Brunswick Laboratory, (1989).

[3] P. Persiani, et. al., "Systems Analysis for Material Control and Accountancy Technology -Analysis of Example Facility for the Workshop on Guides for Material Control and Accountability (MC\&A) Performance Requirements", (Argonne National Laboratory, August, 1989).

4] K.R. Byers, et. al., "Assessment of Alternatives to Correct Inventory Difference Statistical Treatment Deficiencies", NUREG/CR-3256, PNL-4656, (U. S. Nuclear Regulatory Commission, Washington, DC, November 1983).

[5] J. Rivers, "Inventory Difference (ID) Issues",U. S. DOE Memorandum, DP-342.1, Jure 2, 1989 (U. S. Department of Energy, Washington, DC,1989).

I6 R. A. Bari, et al., "Probabilistic Safety Analysis Procedures Guide", NUREG/CR-2815, Vol. 1, Rev. 1, (U. S. Nuclear Regulatory Commission, Washington, DC, August 1985), Section 5.

[7] R. J. Brouns, B. W. Smith, and R. F. Eggers, "Human Error in Nuclear Material Accounting and Control Data", Lournal of the Institute of Nuclear Materials Management XIIL, \#1, (Spring 1984), p.48.

[8] S. C. Suda, "Some Thoughts on Constant and Variable Components of Systematic Error", Lournal of the Institute of Nuclear Materials Management IV, \#1, (Spring 1975), p.41.

(9] R. H. Moore, "Some Thoughts on 'Some Thoughts on Random Errors, Systematic Errors, and Biases'", Lournal of the Institute of Nuclear Materials Management IV \#1, (Spring 1975), p.44. 
[10] K. B. Stewart, "Some Statistical Aspects of Bias Corrections", Lournal of the Institute of Nuclear Materials Management IV, \#2, (Summer 1975), p.20.

[11] A. L. Harkness, "Measurements, Biases and Uncertainties", Lournal of the Institute of Nuclear Materials Management $\underline{\mathrm{V}}, \# 1$, (Spring 1976), p.48.

[12] J. L. Jaech, Statistical Methods in Nuclear Materials Control, TID-26298, (U. S. Atomic Energy Commission, Washington, DC, 1973), p.89.

[13] American National Standards Institute, Inc., "Volume Calibration Techniques for Nuclear Materials Control", ANSI N15.19-1988, (American National Standards Institute, Inc., New York, 1988).

[14] F. E. Jones, "A Tank Volume Calibration Algorithm", Lournal of the Institute of Nuclear Materials Management XIII, \#1, (Spring 1984), p.16.

[15] A. M. Liebetrau, A. S. Goldman, and M. Aparo, "Uncertainty Estimates for Calibration Measurements", Nuclear Safeguards Technology Vol. 2. Proceedings of an International Symposium on Nuclear Materials Safeguards, (International Atomic Energy Agency, Vienna, Austria,1987), p.487.

[16] K. A. Brownlee, Statistical Theory and Mcthodology in Science, Second Edition, (John Wiley and Sons, New York, 1965).

[17] H. Scheffé, The Analysis of Variance, (John Wiley and Sons, New York, 1959).

[18] F. E. Grubbs, "On Estimating Precision of Measuring Instruments and Product Variability", Journal of the American Statistical Association, 42, (1948), p.243.

[19] W. Liggett, "Assessing Errors Related to Characteristics of Items Measured", Lournal of the Institute of Nuclear Materials Management IX, (Spring 1980), p.78.

[20] J. L. Jaech, Statistical Analysis of Measurement Errors, (John Wiley and Sons, New York, 1985). 
[21] K. K. S. Pillay, "Literature Survey", Lournal of the Institute of Nuclear Materials Management XVI, \#4, (July 1988), p.33.

[2] G. A. Hammond and R. L. Hawkins, "The Importance of Understanding Process Holdup - A Department of Energy (DOE) View", Ibid., p.12.

[23] R. J. Brouns and F. P. Roberts, "Control of Sampling Errors", Iournal of the Institute of Nuclear Materials Management, $\mathrm{V}$, (Fall 1976).

[24] L. F. Sears, et al., "Separation Plant Needle Sampler Modifications', AGNS-35900-2.4-74, (Allied-General Nuclear Services, Barnwell, South Carolina, May 1980).

[25] E. P. Shine, "Density Meter Algorithm and System for Estimating Mixing/Sampling Uncertainty", Proceedings of the 27th Annual. Meeting of the Institute of Nuclear Matcrials Management, New Orleans, Louisiana, June 22-25, 1986, Nuclear Materials Management, XV, (1986), p.215.

[26] S. P. Maxwell, "Overview of Density Meters Used at SRP for Sample Validation", Ibid. , p.208.

[27] J. E. Harrell, "Mixing of Fluids in Small-Diameter Tanks by Recirculation", Report Y-1531 (Union Carbide Nuclear Division, Y-12 Plant, Oak Ridge, TN, April 1966 ).

[28] American National Standards Institute, Inc., (ANSI), "Mass Calibration Techniques for Nuclear Material Control", ANSI N15.18-1975, (American National Standards Institute, Inc., New York, 1975).

[29] L. W. Doher and K. G. Gerald, "Application of American National Standards of Calibration Techniques of Bulk Measurements for Nuclear Materials Control", Proceedings of the Second Annual Symposium on Safeguards and Nuclear Materials Management, Edinburgh, Scotland, U.K., 26-28 March, 1980, ESARDA 11, (European Safeguards and Research and Development Association, Brussels, Belgium, 1980), p282.

[30)] J. L. Jaech, "Estimation of Scale Accuracy and Precision: A Case History", Lournal of the Institute of Nuclear Materials Management VII, \#3(Fall 1978), p.81.

[31] Handbook of Tables for Probability and Statistics, William H. Beyer, Editor, 
(CRC Press, Inc., Boca Raton, Florida, 1968), pp.392-3.

[32] T. P. Speed and D. Culpin, "Evaluation of Techniques in Nuclear Materials Accountancy", SIROMATH-84-5, (Siromath Pty. Ltd., Sydney, Australia, May 1985).

[33] J. P. Shipley, "Decision-Directed Materials Accounting Procedures: An Overview", Proceedings of the 22nd Annual Meeting of the Institute of Nuclear Materials Management, San Francisco, California, July 13-15, 1981, Nuclear Materials Management ، X (1981), p.281.

[34] E. Leitner et al., "Sequential Test Procedures for Near-Real-Time Accountancy", Lournal of the Institute of Nuclear Materials Management \& XV (1987), p.29.

[35] R. R. Picard, "Sequential Analysis of Material Balances", Ibid., p.38.

[36] R. R. Picard and J. F. Hafer, "MAWST: Materials Accounting with Sequential Testing", N4/89-111,(Los Alamos National Laboratory, Los Alamos, New Mexico, May 1989).

[37] NUSAC Inc., "Mathematical Derivation of the Automated Materials Accounting Statistics System (AMASS)", (NUSAC Inc., McLean, Virginia, September 15, 1981). 


\section{APPENDIX A}

This appendix contains a derivation of the formulas (2) - (4) in Chapter VI.

Each error appearing in an error model for any of the measurements $m_{i j}$ of equation (1) in Chapter VI is assumed to be associated with some effect $\mathrm{E}$ (which may contain one or more errors) with an associated standard deviation ${ }_{\sigma}^{2}$ The eth error associated with effect $\mathrm{E}$ is designated $\varepsilon_{\mathrm{E}, \mathrm{e}^{\prime}}$ In these terms, and keeping in mind the complicated relationship between errors and measurements depicted in Figure 6.1, the error models can be written as

$$
m_{i j}=\mu_{i j}+\sum_{k} \varepsilon_{E(i j k), e(i j k)}=\mu_{i j}+\Delta_{i j}
$$

(absolute), or

$$
\mathrm{m}_{\mathrm{ij}}=\mu_{\mathrm{ij}}\left(1+\sum_{\mathrm{k}} \varepsilon_{\mathrm{E}(\mathrm{ijk}), \mathrm{e}(\mathrm{ijk})}\right)=\mu_{\mathrm{ij}}\left(1+\Delta_{\mathrm{ij}}\right)
$$

(relative). These can both be expressed as:

$$
m_{i j}=\mu_{i j}+\Gamma_{i j} \Delta_{i j}
$$

where $\Gamma_{i j}=\mu_{i j}$ if the model for the $j$ th measurement of item $i$ is relative, and $\Gamma_{i j}=1$ if it is absolute. Substituting the right-hand expression above into the ID equation [1] in chapter VI,

$$
I D=\sum_{i} s_{i} f_{i}\left(\mu_{i 1}+\Gamma_{i 1} \Delta_{i 1}, \mu_{i 2}+\Gamma_{i 2} \Delta_{i 2}, \ldots\right)
$$

Using the Taylor expansion this becomes, to first order,

$$
\begin{aligned}
I D=\sum_{i} s_{i} f_{i}\left(\mu_{i}\right)+\sum_{i j} s_{i j} f_{i}(j) & \left(\mu_{i}\right) \Gamma_{i j} \Delta_{i j} \\
& \left.=I_{0}+\sum_{i j} s_{i j} f_{i}(j)\left(\mu_{i j}\right) \Gamma_{i j} \Delta_{i j}\right)
\end{aligned}
$$


where the superscript $(j)$ indicates partial derivative with respect to the $j$ th argument, and $\mu_{i}$ represents $\left(\mu_{i 1}, \mu_{i 2}, \ldots\right)$. Using the expression for the error models (1) and (2) above, this becomes

$$
\mathrm{I}_{\mathrm{O}}+\sum_{\mathrm{ijk}} \mathrm{s}_{\mathrm{i}} \mathrm{f}_{\mathrm{i}}^{(\mathrm{j})}\left(\mu_{\mathrm{i}}\right) \Gamma_{\mathrm{ij}} \varepsilon_{\mathrm{E}(\mathrm{ijk}), \mathrm{e}(\mathrm{ijk})}
$$

This sum can be rearranged (or sorted) so that all the like error terms (the subscripts $E(i, j, k)$ and $e(i, j, k)$ appear together.

$$
I_{O}+\sum_{E}\left(\sum_{e \in E}\left[\sum_{(i, j) \in \mathrm{s}} f_{i}(j)\left(\mu_{i}\right) \Gamma_{i j}\right] \varepsilon_{E, e}\right\}
$$

The summation terminology is as explained in V1.A. All the terms in the sum are now uncorrelated. Using

$$
\operatorname{Var}\left(\varepsilon_{\mathrm{E}, \mathrm{e}}\right)=\sigma_{\mathrm{E}}^{2}
$$

the equations $\mathrm{VI}(2)-\mathrm{VI}(4)$ result from summing the variances of uncorrelated terms, and replacing the value $m$ by the observed value $m$. 

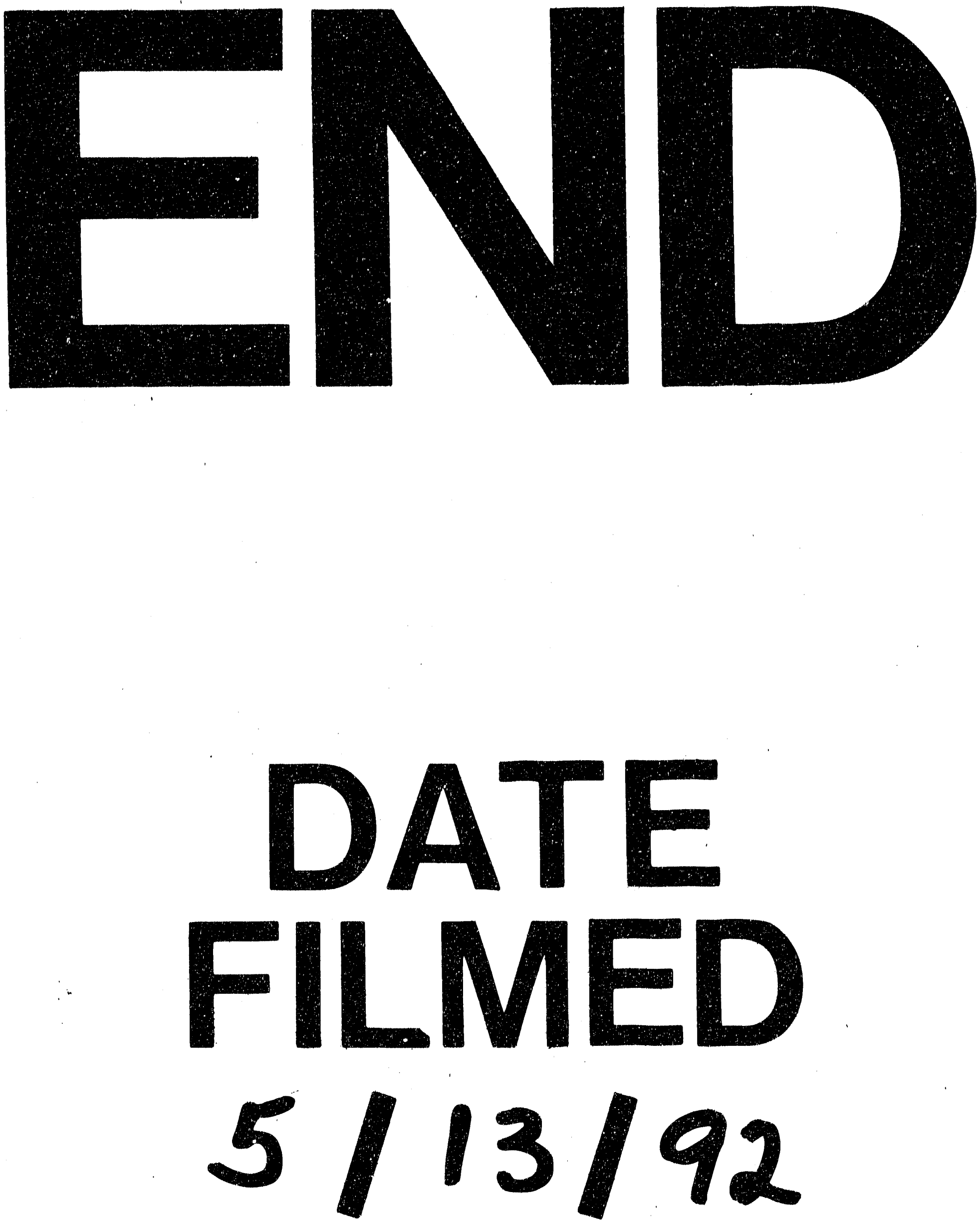
